University of Nebraska - Lincoln

DigitalCommons@University of Nebraska - Lincoln

U.S. Air Force Research

U.S. Department of Defense

2012

\title{
Analysis of three-dimensional fracture mechanics problems: A non-intrusive approach using a generalized finite element method
}

P Gupta

University of Illinois at Urbana-Champaign

JP. Pereira

University of Illinois at Urbana-Champaign

D J. Kim

Kyung Hee University

CA. Duarte

University of Illinois at Urbana-Champaign

T Eason

Air Vehicles Directorate

Follow this and additional works at: http://digitalcommons.unl.edu/usafresearch

Gupta, P; Pereira, J P.; Kim, D J.; Duarte, C A.; and Eason, T, "Analysis of three-dimensional fracture mechanics problems: A nonintrusive approach using a generalized finite element method" (2012). U.S. Air Force Research. 61.

http://digitalcommons.unl.edu/usafresearch/61

This Article is brought to you for free and open access by the U.S. Department of Defense at DigitalCommons@University of Nebraska - Lincoln. It has been accepted for inclusion in U.S. Air Force Research by an authorized administrator of DigitalCommons@University of Nebraska - Lincoln. 


\title{
Analysis of three-dimensional fracture mechanics problems: A non-intrusive approach using a generalized finite element method
}

\author{
P. Gupta a, J.P. Pereira ${ }^{\text {a }}$, D.-J. Kim ${ }^{\text {b }}$, C.A. Duarte ${ }^{\text {a, } *}$, T. Eason ${ }^{c}$ \\ ${ }^{a}$ Department of Civil and Environmental Engineering, University of Illinois at Urbana-Champaign, Newmark Laboratory, 205 North Mathews Avenue, \\ Urbana, IL 61801, USA \\ ${ }^{\mathrm{b}}$ Department of Architectural Engineering, Kyung Hee University, Engineering Building, 1 Sochon-Dong Kihung-Gu, Yongin, Kyunggi-Do 446-701, Republic of Korea \\ ${ }^{\mathrm{c}}$ U.S. Air Force Research Laboratory, Air Vehicles Directorate, WPAFB, Dayton, OH, USA
}

\section{A R T I C L E I N F O}

\section{Article history:}

Received 20 November 2011

Received in revised form 25 March 2012

Accepted 8 April 2012

\section{Keywords:}

Generalized finite element method

Fracture mechanics

Global-local analysis

Multiscale problem

Schur complement

\begin{abstract}
A B S T R A C T
This paper shows that the generalized finite element method with global-local enrichment functions $\left(\mathrm{GFEM}^{\mathrm{gl}}\right)$ can be implemented non-intrusively in existing closed-source FEM software as an add-on module. The $\mathrm{GFEM}^{\mathrm{gl}}$ is based on the solution of interdependent global (structural) and local (crack) scale problems. In the approach presented here, an initial global scale problem is solved by a commercial finite element analysis software, local problems containing 3-D fractures are solved by an $h p$-adaptive GFEM software and an enriched global scale problem is solved by a combination of the FEM and GFEM softwares. The interactions between the solvers are limited to the exchange of load and solution vectors and does not require the introduction of user subroutines to existing FEM software. As a results, the user can benefit from built-in features of available commercial grade FEM software while adding the benefits of the GFEM for this class of problems. Several threedimensional fracture mechanics problems aimed at investigating the applicability and accuracy of the proposed two-solver methodology are presented.
\end{abstract}

(c) 2012 Elsevier Ltd. All rights reserved.

\section{Introduction}

Several industries, ranging from aeronautical to automotive to gas and oil, have the need for simple, robust and accurate computational tools to perform fracture analyses of complex 3-D structures. Finite element methods available in commercial software face several difficulties in solving this class of problems. The discretization of 3-D cracks in complex structures is in general labor intensive and leads to high computational costs, in particular when several crack configurations must be analyzed as in the case of crack growth simulations or multi-site damage analyses. In most finite element software, the analysis is carried out by local remeshing. This may be difficult to automate in cases involving changes in crack topology or surface breaking cracks near complex geometrical features. Mapping of finite element solutions between meshes may also lead to severe loss of accuracy. The global-local or sub-modeling procedure in the FEM can address some of these issues but it may also lead to large errors in computed fracture mechanics quantities [30].

The Generalized or eXtended FEM (G/XFEM) [5] greatly facilitates the discretization of complex 3-D fractures but their accuracy is dependent on the approximation properties of analytically derived enrichment functions. The intersection of a 3-D crack front with the boundary of the domain creates singularities of a type that is dependent on the angle between the crack front and the domain boundary. Thus, the singularity is not necessarily of the type $\sqrt{r}$ as commonly assumed in the G/XFEM. Away from these intersection points, the elastic solution can be presented as an asymptotic series of eigen-pairs

\footnotetext{
* Corresponding author. Tel.: +1 217244 2830; fax: +1 2173333821.

E-mail address: caduarte@illinois.edu (C.A. Duarte).
} 


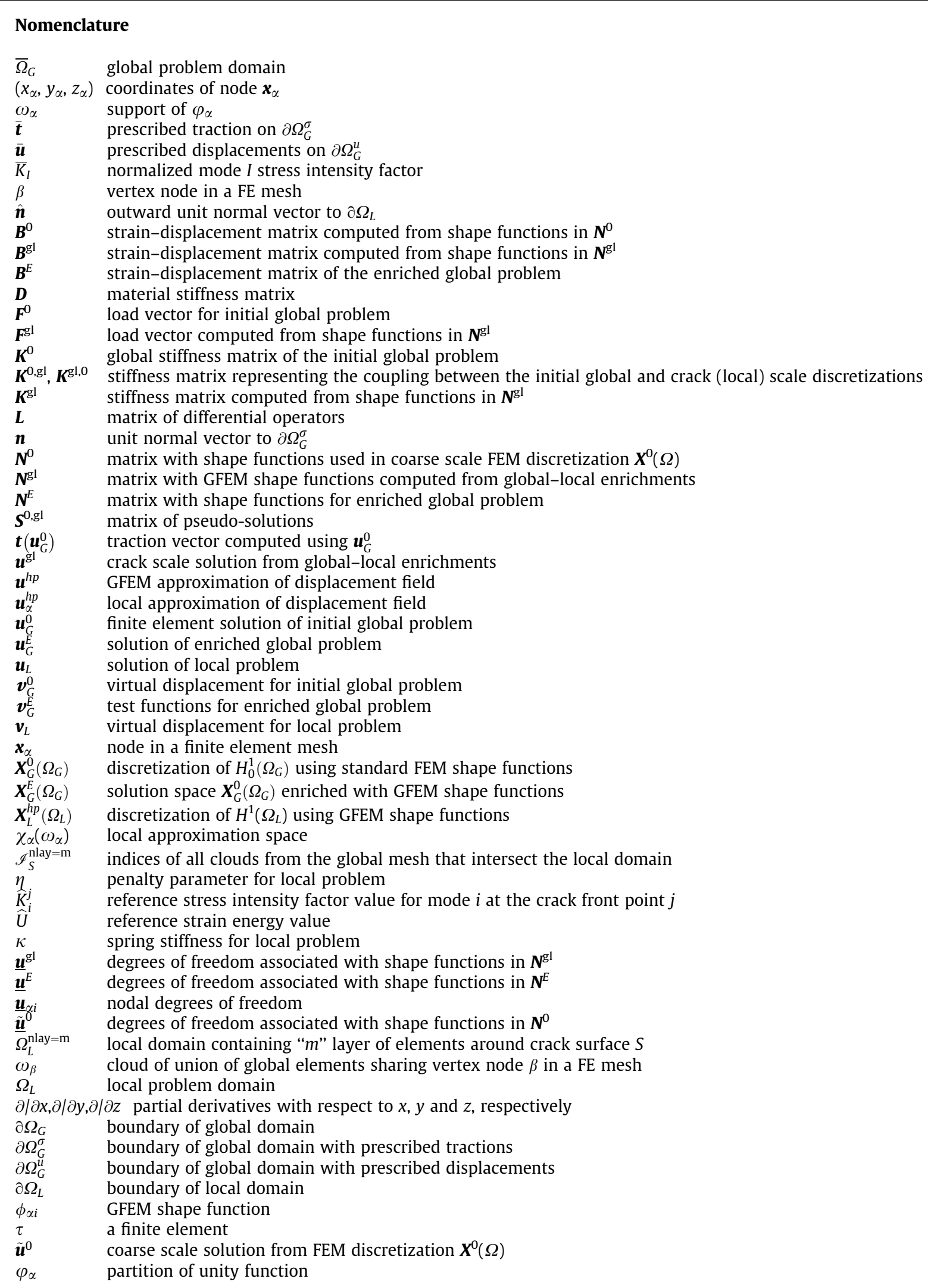




\begin{tabular}{|ll|}
\hline$\widehat{\boldsymbol{F}}^{\mathrm{gl}}$ & load vector for the computation of $\underline{\boldsymbol{u}}^{\mathrm{gl}}$. \\
$\widehat{\boldsymbol{K}}^{\mathrm{gl}}$ & Schur complement of $\boldsymbol{K}^{0}$ \\
$a$ & crack size \\
$D_{L}$ & number of enrichment functions at node \\
$e^{r}$ & relative error in discrete $L^{2}$ norm \\
$e^{r}\left(K_{I}\right)$ & relative error in stress intensity factor $K_{I}$ \\
$e^{r}(U)$ & relative error in strain energy \\
$H_{0}^{1}\left(\Omega_{G}\right)$ & a Hilbert space of functions that are zero on the boundary $\partial \Omega_{G}^{u}$ \\
$h_{\alpha}$ & scaling factor \\
$J$ & Jacobian of the global element across the local boundary where the spring boundary conditions are imposed \\
$K_{i}^{j}$ & computed stress intensity factor value for mode $i$ at the crack front point $j$ \\
$L_{e}$ & maximum element size along the crack front \\
$L_{\alpha i}$ & enrichment function \\
$N$ & number of nodes in finite element mesh \\
$n$ & number of spatial dimensions of the problem \\
$N_{\text {ext }}$ & number of extraction points along the crack front \\
$Q$ & geometry factor for penny shaped crack \\
$r$ & crack radius for surface crack \\
$S$ & crack surface \\
$T$ & tensile traction \\
$U$ & computed strain energy value \\
$V_{0}$ & volume of the master element \\
& \\
\hline
\end{tabular}

(the well known 2-D eigen-pairs) and the associated edge stress intensity functions. However, as opposed to planar elastic problems, each of the eigen-pairs is accompanied by an infinite number of shadow functions with an increasing exponential order [10]. Due to the complex nature of the 3-D solution, 2-D expansions of the elasticity solution are used as enrichment functions for 3-D cracks in finite size domains [15,39,56]. As a consequence, a sufficiently fine mesh must be used around the crack front to achieve acceptable accuracy. In the $h p$-adaptive GFEM presented in [44-46] mesh refinement and enrichment are done in the global discretization of a problem, in the same spirit as in the standard FEM. This offsets some of the attractive features of the G/XFEM and adds computational complexity to their implementation in available FEM software.

In this paper, we use the generalized finite element method with global-local enrichment functions $\left(\mathrm{GFEM}^{\mathrm{gl}}\right)$ proposed in [31] to overcome these limitations. A key feature of this GFEM is that the so-called global-local enrichments are hierarchically added to an existing, possibly coarse, finite element discretization. As a result, it can be implemented non-intrusively in existing closed-source FEM software as an add-on module. In the approach presented here, an initial global scale problem is solved by a commercial finite element analysis software like Abaqus [1], local problems containing 3-D fractures are solved by an $h p$-adaptive GFEM software and an enriched global scale problem is solved by a combination of the FEM and GFEM solvers. The interactions between the solvers are limited to the exchange of load and solution vectors. In this paper, we describe this two-solver methodology and demonstrate that it does not require the introduction of user subroutines to existing FEM software while delivering accurate results on coarse 3-D finite element meshes. As a results, the user can benefit from built-in features of available commercial grade FEM software while adding the benefits of the GFEM for this class of problems. Furthermore, no modifications of available uncracked finite element meshes are needed thus, model preparation times for the proposed two-solver methodology are short. This work demonstrates this non-intrusive algorithm of the GFEM ${ }^{\mathrm{gl}}$ in Abaqus [1]. However, the proposed approach can also be used with other methods able to analyze 3-D fractures effectively, like the boundary element method, and can be implemented in most available FEM softwares.

The proposed two-solver algorithm for the GFEM $^{\text {gl }}$ delivers the same solution and therefore accuracy, as in the single-solver case presented in [31]. The implementation of the single-solver algorithm requires several non-trivial modifications of existing Finite Element solvers such as adaptive mesh refinement and GFEM type shape functions. The proposed algorithm effectively introduce GFEM and $h p$-refinement capabilities to an existing Finite Element Analysis (FEA) platform without any code modifications. The methodology also allows the use of built-in features in both FEA and GFEM softwares. This is a significant advantage from the practical point of view over the single-solver approach presented in [31].

In recent years several researchers have proposed approaches for the implementation of the G/XFEM in commercial FEA software. Bordas et al. [6,7] proposed a combination of an object oriented XFEM C++ code interfaced with EDS-PLM/I-DEAS software for damage tolerance assessment of complex three-dimensional industrial components. Sukumar and Prevost implemented the XFEM in Dynaflow [55]. In Shi et al. [54] a 3-D XFEM toolkit for Abaqus [1] is presented which is developed and validated by a suite of benchmark problems. Their implementation is based on the user element library (UEL) capabilities provided by Abaqus. Gendre et al. [23,24] proposed a non-intrusive methodology with Abaqus based on approximations of the Schur complement of local degrees of freedom. Their methodology focus on problems with localized plasticity. Rank et al. [19,34,51] proposed a methodology to implement hierarchical enrichments like the s-version of the FEM [21] in a 
close-source FEM software. However we are not aware of its actual implementation as of this writing. Nistor et al. [40] implemented the XFEM in an explicit FEM code for dynamic crack analysis using an object oriented framework. Giner et al. $[25,26]$ proposed an Abaqus implementation of 2-D XFEM aimed at the simulation of fretting fracture crack propagation. We can also mention the Abaqus implementations of Lua et al. [36] for curvilinear crack growth and life prediction based on XFEM; Xu and Yuan [64,65] for mixed-mode fatigue crack growth in quasi-brittle materials; and the XFEM toolkit of Shi et al. [53] for automated crack onset and crack growth prediction. These implementations are based on user element libraries added to Abaqus.

The rest of the paper is structured as follows: Section 2 describes the target problems considered. In Sections 3 and 4 the main concepts of the GFEM and the GFEM ${ }^{\mathrm{gl}}$ are summarized. Details of the proposed two-solver methodology are described in Section 5. The versatility and accuracy of the method are demonstrated in Section 7 where several 3-D fracture mechanics problems are solved. Finally we close with concluding remarks in Section 8.

\section{Problem description}

Consider a cracked domain $\bar{\Omega}_{G}=\Omega_{G} \cup \partial \Omega_{G}$ in $\Re^{3}$ as illustrated in Fig. 1 . The boundary is decomposed as $\partial \Omega_{G}=\partial \Omega_{G}^{u} \cup \partial \Omega_{G}^{\sigma}$ with $\partial \Omega_{G}^{u} \cap \partial \Omega_{G}^{\sigma}=\emptyset$. The crack surface $S \subset \partial \Omega_{G}^{\sigma}$ is assumed to be traction-free.

We consider the linear elasticity problem on this domain. The equilibrium equations are given by

$$
\nabla \cdot \boldsymbol{\sigma}=\boldsymbol{0} \quad \text { in } \Omega_{G},
$$

where $\boldsymbol{\sigma}$ is the Cauchy stress tensor. The following boundary conditions are prescribed on $\partial \Omega_{G}$

$$
\boldsymbol{u}=\overline{\boldsymbol{u}} \text { on } \partial \Omega_{G}^{u} \quad \boldsymbol{\sigma} \cdot \boldsymbol{n}=\overline{\boldsymbol{t}} \text { on } \partial \Omega_{G}^{\sigma},
$$

where $\boldsymbol{n}$ is the outward unit normal vector to $\partial \Omega_{G}^{\sigma}$ and $\overline{\boldsymbol{t}}$ and $\overline{\boldsymbol{u}}$ are prescribed tractions and displacements, respectively. Without loss of generality, we assume hereafter that $\overline{\boldsymbol{u}}=\boldsymbol{0}$. The constitutive relations are given by the generalized Hooke's law,

$$
\boldsymbol{\sigma}=\boldsymbol{C}: \boldsymbol{\varepsilon}
$$

where $\boldsymbol{C}$ is Hooke's tensor. The kinematic relations are given by

$$
\boldsymbol{\varepsilon}=\nabla_{s} \boldsymbol{u} \quad \text { in } \Omega_{G},
$$

where $\boldsymbol{\varepsilon}$ is the linear strain tensor and $\nabla_{s}$ is the symmetric part of the gradient operator.

We seek to find an approximation to the solution $\boldsymbol{u}$ of the problem defined by Eqs. (1)-(4) using a FEM implemented in a commercial code like Abaqus [1], and a GFEM implemented in a research code.

\section{The generalized FEM: main concepts}

The generalized finite element method (GFEM) is an instance of the Partition of Unity Method (PUM). Its inception can be traced back to the works of Babuška et al. [2,3,37] and Duarte and Oden [11,16-18,41]. In this class of methods, discretization spaces for a Galerkin method are defined using the concept of a partition of unity. The Lagrangian finite element shape

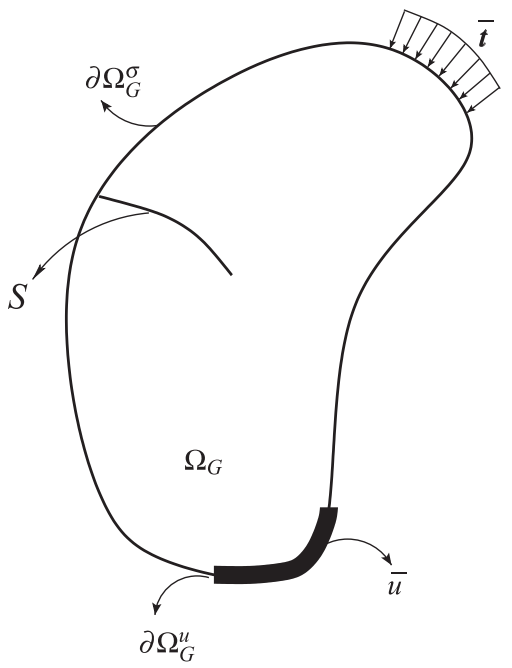

Fig. 1. The linear elastic fracture mechanics problem in 3-D 
functions $\varphi_{\alpha}, \alpha=1, \ldots, N$, in a finite element mesh with $N$ nodes constitute a partition of unity, i.e., $\sum_{\alpha=1}^{N} \varphi_{\alpha}(\boldsymbol{x})=1$ for all $\boldsymbol{x}$ in a domain $\Omega$ covered by the finite element mesh.

The GFEM denotes a PUM with the partition of unity provided by Lagrangian finite element shape functions. The same method is also known as the eXtended FEM (XFEM) [4,38]. A recent review of Generalized/eXtended FEMs along with a brief history of their developments can be found in [5,22]. The Finite Cover Method [59] and the Manifold method [52] also share many similarities with the GFEM.

A shape function, $\phi_{\alpha i}$, in the GFEM is computed from the product of a Lagrangian shape function, $\varphi_{\alpha}$, and an enrichment function, $L_{\alpha i}$,

$$
\phi_{\alpha i}(\boldsymbol{x})=\varphi_{\alpha}(\boldsymbol{x}) L_{\alpha i}(\boldsymbol{x}) \quad(\text { no summation on } \alpha)
$$

where $\alpha$ is a node in the finite element mesh. Linear combinations of the GFEM shape functions $\phi_{\alpha i}, \alpha=1, \ldots, N$, can represent exactly any enrichment function $L_{\alpha i}[11,17]$. Fig. 2 outlines the construction of GFEM shape functions.

Several enrichment functions can be hierarchically added to any node $\alpha$ in a finite element mesh. Thus, if $D_{L}$ is the number of enrichment functions at node $\alpha$, the GFEM approximation, $\boldsymbol{u}^{h p}$, of a vector field $\boldsymbol{u}$ can be written as

$$
\boldsymbol{u}^{h p}(\boldsymbol{x})=\sum_{\alpha=1}^{N} \sum_{i=1}^{D_{L}} \underline{\boldsymbol{u}}_{\alpha i} \phi_{\alpha i}(\boldsymbol{x})=\sum_{\alpha=1}^{N} \sum_{i=1}^{D_{L}} \underline{\boldsymbol{u}}_{\alpha i} \varphi_{\alpha}(\boldsymbol{x}) L_{\alpha i}(\boldsymbol{x})=\sum_{\alpha=1}^{N} \varphi_{\alpha}(\boldsymbol{x}) \sum_{i=1}^{D_{L}} \underline{\boldsymbol{u}}_{\alpha i} L_{\alpha i}(\boldsymbol{x})=\sum_{\alpha=1}^{N} \varphi_{\alpha}(\boldsymbol{x}) \boldsymbol{u}_{\alpha}^{h p}(\boldsymbol{x})
$$

where $\underline{\boldsymbol{u}}_{\alpha i}, \alpha=1, \ldots, N, i=1, \ldots, D_{L}$, are nodal degrees of freedom and $\boldsymbol{u}_{\alpha}^{h p}(\boldsymbol{x})$ is an approximation of $\boldsymbol{u}$ defined on $\omega_{\alpha}=\left\{\boldsymbol{x} \in \Omega: \varphi_{\alpha}(\boldsymbol{x}) \neq 0\right\}$, the support of the partition of unity function $\varphi_{\alpha}$. In the case of a finite element partition of unity, the support $\omega_{\alpha}$ (often called a cloud) is given by the union of the finite elements sharing a vertex node $\boldsymbol{x}_{\alpha}$ [14]. The equation above shows that the global GFEM approximation $\boldsymbol{u}^{h p}(\boldsymbol{x})$ is built by pasting together cloud-wise approximations $\boldsymbol{u}_{\alpha}^{h p}, \alpha=1, \ldots, N$, using a partition of unity. This approximation can be used, for example, in a variational principle like the Principle of Virtual Work to find approximate solutions to boundary and initial value problems.

The cloud-wise approximations $\boldsymbol{u}_{\alpha}^{h p}, \alpha=1, \ldots, N$, belong to spaces $\chi_{\alpha}\left(\omega_{\alpha}\right)=\operatorname{span}\left\{L_{\alpha i}\right\}_{i=1}^{D_{L}}$ defined on the supports $\omega_{\alpha}$, $\alpha=1, \ldots, N$. A-priori knowledge about the behavior of the function $\boldsymbol{u}$ over the cloud $\omega_{\alpha}$ is used when selecting enrichment or basis functions for a particular space $\chi_{\alpha}\left(\omega_{\alpha}\right)$. We refer to $[8,14,15,27,39,44,45,56,57]$ and the references therein, for details on the selection of these functions for the case 3-D linear elastic fracture mechanics problems like those considered in this paper.

In the absence of cracks, polynomial enrichment functions can be used. Consider the case of a linear partition of unity provided by four-node tetrahedral elements (TET4). Let $\tau$ denote an element with nodes $\alpha=1,2,3,4$. Quadratic GFEM shape functions for this element are given by [14]

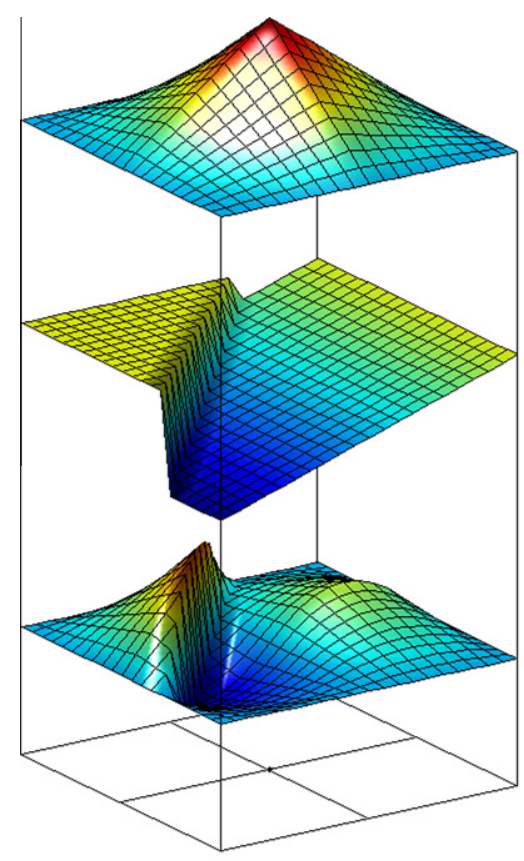

(a)

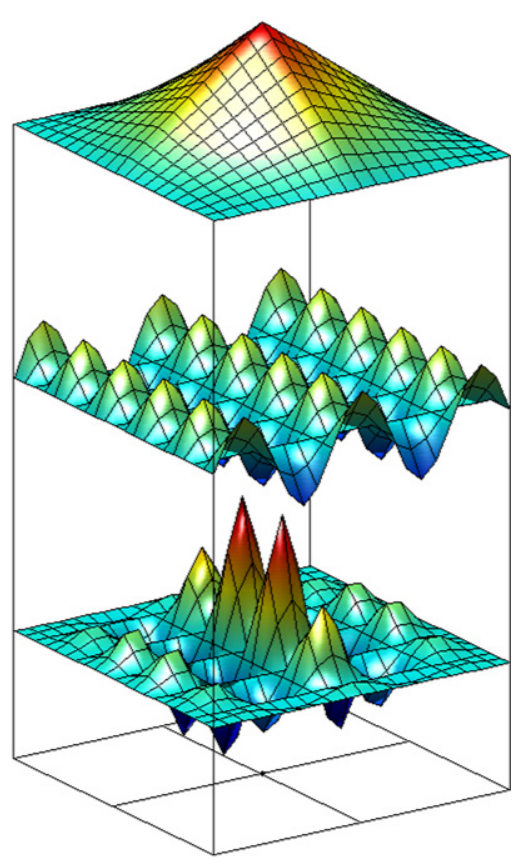

(b)

Fig. 2. Construction of a generalized FEM shape function using a discontinuous (a) and an oscillatory enrichment (b). Here, $\varphi_{\alpha}$ are the functions at the top, the enrichment functions, $L_{\alpha i}$, are the functions in the middle, and the generalized FE shape functions, $\phi_{\alpha i}$, are the resulting bottom functions. 


$$
\varphi_{\alpha} \times\left\{1, \frac{x-x_{\alpha}}{h_{\alpha}}, \frac{y-y_{\alpha}}{h_{\alpha}}, \frac{z-z_{\alpha}}{h_{\alpha}}\right\} \quad \alpha=1,2,3,4
$$

where $\left(x_{\alpha}, y_{\alpha}, z_{\alpha}\right)$ are the coordinates of node $\boldsymbol{x}_{\alpha}$ and $h_{\alpha}$ is an scaling factor [14]. A comparison between the performance of this element and standard ten-node quadratic tetrahedral elements (TET10) is presented in [14]. Both types of elements are used in the numerical experiments presented in Section 7. Cubic and higher order GFEM shape functions are built using the same procedure [14].

If the enrichment functions $L_{\alpha 1}=1, \alpha=1, \ldots, N$, the GFEM approximation given by (6) can be written as

$$
\boldsymbol{u}^{h p}(\boldsymbol{x})=\sum_{\alpha=1}^{N} \sum_{i=1}^{D_{L}} \underline{\boldsymbol{u}}_{\alpha i} \varphi_{\alpha}(\boldsymbol{x}) L_{\alpha i}(\boldsymbol{x})=\sum_{\alpha=1}^{N} \varphi_{\alpha}(\boldsymbol{x})\left[\underline{\boldsymbol{u}}_{\alpha 1}+\sum_{i=2}^{D_{L}} \underline{\boldsymbol{u}}_{\alpha i} L_{\alpha i}(\boldsymbol{x})\right]=\underbrace{\sum_{\alpha=1}^{N} \underline{\boldsymbol{u}}_{\alpha 1} \varphi_{\alpha}(\boldsymbol{x})}_{\text {standard FEM approx. }}+\underbrace{\sum_{\alpha=1}^{N} \sum_{i=2}^{D_{L}} \underline{\boldsymbol{u}}_{\alpha i} \varphi_{\alpha}(\boldsymbol{x}) L_{\alpha i}(\boldsymbol{x})}_{\text {GFEM enrichment }}
$$

This clearly shows the hierarchical nature of a GFEM approximation. This property enables the solution of problems involving more than one spatial scale of interest using a standard FEM and a GFEM solver as described later on in this paper.

\section{Solution of fracture mechanics problems using global-local enrichments}

In $[12,13,31]$ we present a global-local approach to build enrichment functions for the generalized FEM. This approach is based on the solution of the interdependent global and local scale problems. The local problems focus on the solution of a boundary value problems defined in the neighborhood of 3-D cracks while the global problem approximates the macro or structural scale behavior of an uncracked domain. This so-called GFEM with global-local enrichment functions is hereafter denoted $\mathrm{GFEM}^{\mathrm{gl}}$. In the formulation presented in this section, the global problem is solved by a commercially available FE software while local problems are solved using a GFEM software capable of performing automatic mesh refinement and enrichment, hereafter denoted $h p$-GFEM $[44,45]$. The interactions between the two solvers are described in Section 5 . The proposed methodology enables modeling of small cracks on coarse, uncracked, global meshes.

\subsection{Formulation of structural scale problem}

Let $\boldsymbol{u}_{G}^{0}$ denote the standard FEM solution of the problem described in Section 2 but without cracks. This is hereafter denoted as the initial global problem. The approximation $\boldsymbol{u}_{G}^{0}$ is the solution of the following problem:

Find $\boldsymbol{u}_{G}^{0} \in \boldsymbol{X}_{G}^{0}\left(\Omega_{G}\right) \subset H_{0}^{1}\left(\Omega_{G}\right)$ such that, $\forall \boldsymbol{v}_{G}^{0} \in \boldsymbol{X}_{G}^{0}\left(\Omega_{G}\right)$

$$
\int_{\Omega_{G}} \boldsymbol{\sigma}\left(\boldsymbol{u}_{G}^{0}\right): \boldsymbol{\varepsilon}\left(\boldsymbol{v}_{G}^{0}\right) d \boldsymbol{x}=\int_{\partial \Omega_{G}^{\sigma}} \overline{\boldsymbol{t}} \cdot \boldsymbol{v}_{G}^{0} d \boldsymbol{s}
$$

where $\boldsymbol{X}_{G}^{0}\left(\Omega_{G}\right)$ is a discretization of $H_{0}^{1}\left(\Omega_{G}\right)$, a Hilbert space of functions that are zero on the boundary $\partial \Omega_{G}^{u}$. The discretization is defined using standard FEM shape functions available in a commercial software like Abaqus [1]. Accurate solutions can be computed using quasi uniform meshes like the one shown in Fig. 3 since no cracks are considered in the initial global problem.

\subsection{Formulation of crack scale local problem}

In the GFEM ${ }^{\mathrm{gl}}$, a local boundary value problem is defined in a neighborhood $\Omega_{L}$ of each crack in the global domain $\Omega_{G}$. These local problems are solved using the solution of the initial global problem $\boldsymbol{u}_{G}^{0}$ as boundary conditions on $\partial \Omega_{L} \backslash\left(\partial \Omega_{L} \cap \partial \Omega_{G}\right)$, the portion of the local boundary that does not intersect the boundary of the global problem. The statement of the principle of virtual work for a local problem is given by.

Find $\boldsymbol{u}_{L} \in \boldsymbol{X}_{L}^{h p}\left(\Omega_{L}\right) \subset H^{1}\left(\Omega_{L}\right)$ such that, $\forall \boldsymbol{v}_{L} \in \boldsymbol{X}_{L}^{h p}\left(\Omega_{L}\right)$

$$
\begin{aligned}
\int_{\Omega_{L}} \boldsymbol{\sigma}\left(\boldsymbol{u}_{L}\right) & : \boldsymbol{\varepsilon}\left(\boldsymbol{v}_{L}\right) d \boldsymbol{x}+\eta \int_{\partial \Omega_{L} \cap \partial \Omega_{G}^{u}} \boldsymbol{u}_{L} \cdot \boldsymbol{v}_{L} d \boldsymbol{s}+\kappa \int_{\partial \Omega_{L} \backslash\left(\partial \Omega_{L} \cap \partial \Omega_{G}\right)} \boldsymbol{u}_{L} \cdot \boldsymbol{v}_{L} d \boldsymbol{s} \\
& =\int_{\partial \Omega_{L} \cap \partial \Omega_{G}^{\sigma}} \overline{\boldsymbol{t}} \cdot \boldsymbol{v}_{L} d \boldsymbol{s}+\int_{\partial \Omega_{L} \backslash\left(\partial \Omega_{L} \cap \partial \Omega_{G}\right)}\left(\boldsymbol{t}\left(\boldsymbol{u}_{G}^{0}\right)+\kappa \boldsymbol{u}_{G}^{0}\right) \cdot \boldsymbol{v}_{L} d \boldsymbol{s}
\end{aligned}
$$

where $\boldsymbol{X}_{L}^{h p}\left(\Omega_{L}\right)$ is a discretization of $H^{1}\left(\Omega_{L}\right)$ using the GFEM shape functions presented in $[31,44,45]$ combined with automatic mesh refinement and enrichment. This $\mathrm{hp}$-GFEM allows the solution of local problems using meshes that do not fit the crack surface as illustrated in Figs. 6 and 7. Details can be found in Section 3.2 of [44]. The partition of unity used in the local problems is provided by linear TET4 elements (see also Section 6).

The parameter $\eta$ in (10) is a penalty parameter based on the Young's modulus and the Jacobian of the elements with a face on the portion of the local boundary with prescribed displacements. The penalty method is used in our implementation of the $h p$-GFEM due to its simplicity and generality. Other methods to impose Dirichlet boundary conditions can be used as well. 


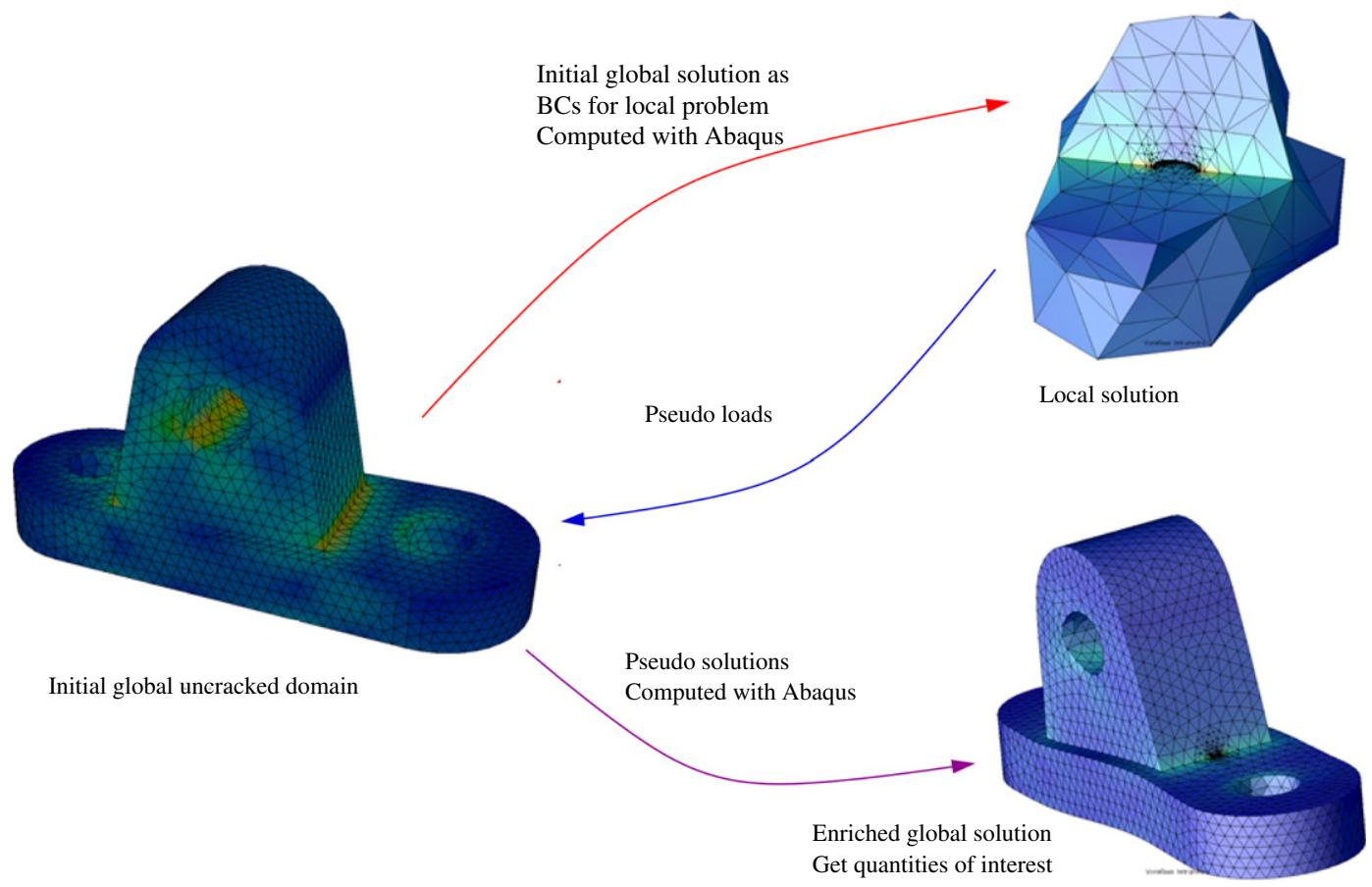

Fig. 3. Illustration of the non-intrusive two-solver algorithm for the analysis of three-dimensional fractures. The global problem is solved by a general purpose FE software while a GFEM code handles the approximation in the neighborhood of fractures.

A key aspect of the GFEM ${ }^{g 1}$ is the use of the global solution $\boldsymbol{u}_{G}^{0}$ to prescribe boundary conditions on $\partial \Omega_{L} \backslash\left(\partial \Omega_{L} \cap \partial \Omega_{G}\right)$. The traction vector, $\boldsymbol{t}\left(\boldsymbol{u}_{G}^{0}\right)$, that appears in the integral over $\partial \Omega_{L} \backslash\left(\partial \Omega_{L} \cap \partial \Omega_{G}\right)$ is computed from the coarse scale solution using Cauchy's relation, i.e.,

$$
\boldsymbol{t}\left(\boldsymbol{u}_{G}^{0}\right)=\hat{\boldsymbol{n}} \cdot \boldsymbol{\sigma}\left(\boldsymbol{u}_{G}^{0}\right)=\hat{\boldsymbol{n}} \cdot\left(\boldsymbol{C}: \boldsymbol{\varepsilon}\left(\boldsymbol{u}_{G}^{0}\right)\right)
$$

with $\hat{\boldsymbol{n}}$ the outward unit normal vector to $\partial \Omega_{L}$. The parameter $\kappa$ is a spring stiffness defined on $\partial \Omega_{L} \backslash\left(\partial \Omega_{L} \cap \partial \Omega_{G}\right)$.

Different types of boundary conditions can be prescribed on $\partial \Omega_{L} \backslash\left(\partial \Omega_{L} \cap \partial \Omega_{G}\right)$ depending on the selected spring stiffness $\kappa$ [31]. If the value of $\kappa$ is set to zero Neumann boundary conditions are enforced. If, however, a large value is assigned to $\kappa$, displacement boundary conditions are prescribed. Intermediate values for $\kappa$ lead to spring or Robin boundary conditions. Numerical experiments presented in [31] show that any value of $\kappa$ comparable to, or larger than the stiffness of the body is acceptable and provides global-local enrichment functions with good approximation properties. The following spring stiffness $\kappa$ is recommended for linear elasticity problems in [31]

$$
\kappa=\frac{E}{\sqrt[n]{V_{0} J}}
$$

where $E$ is the Young's modulus, $n$ is the number of spatial dimensions of the problem, $V_{0}$ is the volume of the master element and $J$ is the Jacobian of the global element across the local boundary where the spring boundary conditions are imposed. Details about the effect of different boundary condition types and spring stiffness values can be found in [31].

The local problem defined in (10) can be computed by a research $h p$-GFEM code while the initial global solution $\boldsymbol{u}_{G}^{0}$ can be computed by a commercial FE software. This is just an example of the well known global-local analysis [20] with local solutions computed by a GFEM instead of the standard FEM. As such, the local solution may have large errors due to the use of inexact boundary conditions on $\partial \Omega_{L} \backslash\left(\partial \Omega_{L} \cap \partial \Omega_{G}\right)$. It turns out that the local solution $\boldsymbol{u}_{L}$ can be used as enrichment functions for the global problem while not requiring any modifications to existing commercial FE software. These ideas are explored in the next sessions. Hereafter, the procedure used to compute $\boldsymbol{u}_{L}$ is denoted by GL-FEM.

\subsection{Global-local enrichment functions and enriched global problem}

The solution $\boldsymbol{u}_{L}$ of the local problem defined in (10) can be used to build generalized FEM shape functions for the coarse global mesh. Eq. (5) is used with the partition of unity function, $\varphi_{\alpha}$, provided by the global finite element mesh and the enrichment function given by $\boldsymbol{u}_{L}$, i.e.,

$$
\boldsymbol{\phi}_{\alpha}(\boldsymbol{x})=\varphi_{\alpha}(\boldsymbol{x}) \boldsymbol{u}_{L}(\boldsymbol{x})
$$


Hereafter, $\boldsymbol{u}_{L}$ is denoted a global-local enrichment function and the global problem enriched with these functions is denoted an enriched global problem. The formulation of this problem is given by

Find $\boldsymbol{u}_{G}^{E} \in \boldsymbol{X}_{G}^{E}\left(\Omega_{G}\right) \subset H_{0}^{1}\left(\Omega_{G}\right)$ such that, $\forall \boldsymbol{v}_{G}^{E} \in \boldsymbol{X}_{G}^{E}\left(\Omega_{G}\right)$

$$
\int_{\Omega_{G}} \boldsymbol{\sigma}\left(\boldsymbol{u}_{G}^{E}\right): \boldsymbol{\varepsilon}\left(\boldsymbol{v}_{G}^{E}\right) d \boldsymbol{x}=\int_{\partial \Omega_{G}^{\sigma}} \overline{\boldsymbol{t}} \cdot \boldsymbol{v}_{G}^{E} d \boldsymbol{s}
$$

where $\boldsymbol{X}_{G}^{E}\left(\Omega_{G}\right)$ is the space $\boldsymbol{X}_{G}^{0}\left(\Omega_{G}\right)$ enriched with GFEM functions (13). The enriched global problem (14) can be solved on the same global mesh used in the computation of the initial global solution $\boldsymbol{u}_{G}^{0}$ since GFEM functions (13) are defined using the coarse scale partition of unity functions $\varphi_{\alpha}$.

Extensive numerical and mathematical analysis presented in $[13,28,31]$ demonstrate that the accuracy of $\boldsymbol{u}_{G}^{E}$ is close to that provided by a direct simulation of the problem with cracks discretized in the global problem. This can be explained by the fact that the local solution $\boldsymbol{u}_{L}$ can approximate well the type of singularity at the crack front even though it may have a large error due to inexact boundary conditions applied to the local problem [28]. This also explains why the GFEM ${ }^{\mathrm{gl}}$ is in general much more accurate than the GL-FEM.

\section{Solution of enriched global problem using a two-solver methodology}

Global-local enrichments add only three degrees of freedom to a node of the global problem when solving a 3-D elasticity problem, regardless of the number of degrees of freedom of the local problem (several thousands in general). Furthermore, enrichments in the GFEM are hierarchical as demonstrated using (8). These two properties of the GFEM ${ }^{\mathrm{gl}}$ are explored in this section. We show that the enriched global problem (14) can be solved by a non-intrusive interaction between Abaqus and a GFEM code. In this section, all quantities are from the global problem and the subscript $G$ is dropped.

The solution $\boldsymbol{u}^{E}$ of the enriched global problem (14) belongs to $\boldsymbol{X}^{E}(\Omega)$ and therefore can be written as:

$$
\underbrace{\tilde{\boldsymbol{u}}^{E}=}_{\text {uarse scale(FEM) }}+\underbrace{\tilde{\boldsymbol{u}}^{\mathrm{gl}}}_{\text {crack scale(GFEM) }}=\boldsymbol{N}^{E} \underline{\boldsymbol{u}}^{E}=\left[\boldsymbol{N}^{0} \boldsymbol{N}^{\mathrm{gl}}\right]\left[\begin{array}{l}
\underline{\tilde{\boldsymbol{u}}}^{0} \\
\underline{\boldsymbol{u}}^{\mathrm{gl}}
\end{array}\right]
$$

where $\boldsymbol{N}^{0}$ is a matrix with shape functions used in the coarse scale FEM discretization $\boldsymbol{X}^{0}(\Omega), \boldsymbol{N}^{\mathrm{gl}}$ has the GFEM shape functions defined in (13), $\underline{\tilde{\boldsymbol{u}}}^{0}$ are degrees of freedom associate with shape functions in $\boldsymbol{N}^{0}$ and $\underline{\boldsymbol{u}}^{\text {gl }}$ are degrees of freedom associated with (hierarchical) global-local enrichment functions in $\mathbf{N}^{\mathrm{gl}}$. Using this two-scale decomposition of the displacement field, the strain-displacement matrix of the enriched global problem can be written as

$$
\boldsymbol{B}^{E}=\boldsymbol{L}\left[\boldsymbol{N}^{0} \boldsymbol{N}^{\mathrm{gl}}\right]=\left[\boldsymbol{B}^{0} \boldsymbol{B}^{\mathrm{gl}}\right]
$$

where

$$
\boldsymbol{L}=\left[\begin{array}{lll}
\partial / \partial x & 0 & 0 \\
0 & \partial / \partial y & 0 \\
0 & 0 & \partial / \partial z \\
\partial / \partial y & \partial / \partial x & 0 \\
0 & \partial / \partial z & \partial / \partial y \\
\partial / \partial z & 0 & \partial / \partial x
\end{array}\right]
$$

This leads to the following decomposition of the stiffness matrix and load vector of the enriched global problem

$$
\left[\begin{array}{ll}
\boldsymbol{K}^{0} & \boldsymbol{K}^{0, \mathrm{gl}} \\
\boldsymbol{K}^{\mathrm{gl}, 0} & \boldsymbol{K}^{\mathrm{gl}}
\end{array}\right]\left[\begin{array}{l}
\underline{\tilde{\boldsymbol{u}}}^{0} \\
\underline{\boldsymbol{u}}^{\mathrm{gl}}
\end{array}\right]=\left[\begin{array}{l}
\boldsymbol{F}^{0} \\
\boldsymbol{F}^{\mathrm{gl}}
\end{array}\right]
$$

where

$$
\boldsymbol{K}^{0}=\int_{\Omega_{G}}\left(\boldsymbol{B}^{0}\right)^{T} \boldsymbol{D} \boldsymbol{B}^{0} d \Omega
$$

is the global stiffness matrix of the initial global problem (9). This matrix can be computed by a standard FE software like Abaqus [1]. The matrix

$$
\boldsymbol{K}^{0, \mathrm{gl}}=\left(\boldsymbol{K}^{\mathrm{gl}, 0}\right)^{T}=\int_{\Omega_{L}}\left(\boldsymbol{B}^{0}\right)^{T} \boldsymbol{D} \boldsymbol{B}^{\mathrm{gl}} d \Omega
$$

represents the coupling between the initial global and crack (local) scale discretizations, and

$$
\boldsymbol{K}^{\mathrm{gl}}=\int_{\Omega_{L}}\left(\boldsymbol{B}^{\mathrm{gl}}\right)^{\mathrm{T}} \boldsymbol{D} \boldsymbol{B}^{\mathrm{gl}} d \Omega
$$


represents the global stiffness associated with GFEM shape functions (13). The initial global problem stiffness matrix is nested in the enriched global problem stiffness matrix since the GFEM shape functions (13) are hierarchically added to the initial problem discretization. Since the dimension of $\underline{u}^{\mathrm{gl}}$ is, for practical problems, much smaller than the dimension of $\underline{\tilde{\boldsymbol{u}}}^{0}$, the system of Eq. (17) can be efficiently solved through static condensation [20] of the degrees of freedom $\underline{\boldsymbol{u}}^{\mathrm{gl}}$. This process can be implemented non-intrusively using a commercial software like Abaqus [1] and a GFEM code. This two-solver algorithm is described below.

\subsection{Static condensation of global-local degrees of freedom}

From the first equation in (17)

$$
\begin{aligned}
\boldsymbol{K}^{0} & \tilde{\boldsymbol{u}}^{0}=\boldsymbol{F}^{0}-\boldsymbol{K}^{0, \mathrm{gl}} \underline{\boldsymbol{u}}^{\mathrm{gl}} \\
\underline{\tilde{\boldsymbol{u}}}^{0} & =\left(\boldsymbol{K}^{0}\right)^{-1}\left[\boldsymbol{F}^{0}-\boldsymbol{K}^{0, \mathrm{gl}} \underline{\boldsymbol{u}}^{\mathrm{gl}}\right] \\
\underline{\tilde{\boldsymbol{u}}}^{0} & =\left(\boldsymbol{K}^{0}\right)^{-1} \boldsymbol{F}^{0}-\left(\boldsymbol{K}^{0}\right)^{-1} \boldsymbol{K}^{0, \mathrm{gl} \mathrm{u}} \underline{\boldsymbol{u}}^{\mathrm{gl}} \\
& =\underline{\boldsymbol{u}}^{0}-\boldsymbol{S}^{0, \mathrm{gl} \mathrm{u}} \underline{\boldsymbol{u}}^{\mathrm{gl}}
\end{aligned}
$$

where $\underline{\underline{u}}^{0}$ is the solution vector of the initial global problem and

$$
\begin{aligned}
& \boldsymbol{S}^{0, \mathrm{gl}}=\left(\boldsymbol{K}^{0}\right)^{-1} \boldsymbol{K}^{0, \mathrm{gl}} \underbrace{\boldsymbol{S}^{0, \mathrm{gl}}}_{\text {pseudo solutions }}=\underbrace{\boldsymbol{K}^{0, \mathrm{gl}}}_{\text {pseudo loads }} .
\end{aligned}
$$

The columns of matrix $\boldsymbol{S}^{\mathbf{0 , g 1}}$ represent pseudo solutions computed by the FEM software through forward and backward substitutions on $\boldsymbol{K}^{0}$, which has already been factorized when solving the (large) initial global problem. The number of pseudo solutions is equal to the number of global-local enrichment degrees of freedom. Thus, the computation of $\boldsymbol{S}^{0, \mathrm{gl}}$ can be performed by the FEM software at a low computational cost. The pseudo loads, $\boldsymbol{K}^{0, \mathrm{gl}}$ are computed by the GFEM software using (18) and sent to the FEA software via, for example, data files. This process is illustrated in Fig. 3.

From the second equation in (17) and the above

$$
\begin{aligned}
& \boldsymbol{K}^{\mathrm{gl}} \underline{\boldsymbol{u}}^{\mathrm{gl}}=\boldsymbol{F}^{\mathrm{gl}}-\boldsymbol{K}^{\mathrm{gl}, 0} \tilde{\boldsymbol{u}}^{0} \\
& \boldsymbol{K}^{\mathrm{gl}} \underline{\boldsymbol{u}}^{\mathrm{gl}}=\boldsymbol{F}^{\mathrm{gl}}-\boldsymbol{K}^{\mathrm{gl}, 0}\left[\underline{\boldsymbol{u}}^{0}-\boldsymbol{S}^{0, \mathrm{gl}} \underline{\boldsymbol{u}}^{\mathrm{gl}}\right]
\end{aligned}
$$

Thus

$$
\underbrace{\left[\boldsymbol{K}^{\mathrm{gl}}-\boldsymbol{K}^{\mathrm{gl}, 0} \boldsymbol{S}^{0, \mathrm{gl}}\right]}_{\widehat{\boldsymbol{K}}^{\mathrm{gl}}} \underline{\boldsymbol{u}}^{\mathrm{gl} \mathrm{l}}=\underbrace{\boldsymbol{F}^{\mathrm{gl}}-\boldsymbol{K}^{\mathrm{gl}, 0} \underline{\boldsymbol{u}}^{0}}_{\widehat{\boldsymbol{F}}^{\mathrm{gl}}}
$$

The global-local degrees of freedom, $\underline{u}^{\mathrm{gl}}$, can be computed from the solution of the following system of equations

$$
\widehat{\boldsymbol{K}}^{\mathrm{gl}} \underline{\boldsymbol{u}}^{\mathrm{gl}}=\widehat{\boldsymbol{F}}^{\mathrm{gl}} .
$$

This small system can be solved by the GFEM software. All that it requires is the computation of matrix $\widehat{\mathbf{K}}^{\text {gl }}$ and right hand side $\widehat{\boldsymbol{F}}^{\text {gl }}$. These, in turn, can be computed using the pseudo solutions $\boldsymbol{S}^{\text {ogl }}$ provided by the FEM software. Note that $\widehat{\boldsymbol{K}}^{\text {gl }}$ is just the Schur complement of $\boldsymbol{K}^{0}$.

Finally, the coarse scale degrees of freedom and the solution of the enriched global problem are given by

$$
\begin{aligned}
& \underline{\tilde{\boldsymbol{u}}}^{0}=\underline{\boldsymbol{u}}^{0}-\boldsymbol{S}^{\mathrm{ogl} \mathrm{g}} \underline{\boldsymbol{u}}^{\mathrm{gl}} \\
& \boldsymbol{u}^{E}=\tilde{\boldsymbol{u}}^{0}+\boldsymbol{u}^{\mathrm{gl}}=\left[\boldsymbol{N}^{0} \boldsymbol{N}^{\mathrm{gl}}\right]\left[\begin{array}{l}
\tilde{\tilde{\boldsymbol{u}}}^{0} \\
\underline{\boldsymbol{u}}^{\mathrm{gl}}
\end{array}\right]
\end{aligned}
$$

As demonstrated above, the computation of $\boldsymbol{u}^{E}$ involves exchange of pseudo loads and pseudo solutions between GFEM and FEA softwares. Fig. 3 illustrates the implementation of the algorithm using the FE software Abaqus and GFEM research code ISET (Illinois Scientific and Engineering Toolkit). Some implementation details of this so-called two-solver methodology are discussed next.

\subsection{Two-solver algorithm}

The implementation of the two-solver algorithm for the GFEM ${ }^{\mathrm{gl}}$ using static condensation can be summarized as follows: 
1. Solve the initial global problem using available FE software. Cracks are not discretized in this problem and coarse meshes able to capture only the global behavior of the problem can be used;

2. Dump to a file the solution vector $\underline{\boldsymbol{u}}^{0}$ computed in the previous step; convert it to the format used by the GFEM software;

3. Read $\underline{\boldsymbol{u}}^{0}$ in GFEM code; create and solve local problem(s) (10) using the $h p$-GFEM [44]. A local problem discretization is created fully automatically using the geometric description of the crack surface [44] and a copy of the global mesh close to the crack (cf. Section 7.1 and Section A.1 of [13]);

4. Compute in the GFEM code and dump to a file pseudo-loads $\boldsymbol{K}^{0, \mathrm{gl}}$ defined in (18); convert them to the format used by the FE software;

5. Read $\boldsymbol{K}^{0, \mathrm{gl}}$ in the FE code and compute pseudo-solutions $\boldsymbol{S}^{0, \mathrm{gl}}$ using (20); dump $\boldsymbol{S}^{0, \mathrm{gl}}$ to a file and convert them to the format used by the GFEM software;

6. Read $\boldsymbol{S}^{0, g l}$ in the GFEM code and compute the solution of the enriched global problem, $\boldsymbol{u}^{E}$, using (21)-(23).

The two-solver algorithm is illustrated in the flowchart shown in Fig. 4. It is clear from the above that the algorithm does not require the introduction of any user subroutines in the FE software as it involves only the exchange of solution and load vectors between two codes. Data file format conversion can be implemented using a stand-alone software. The above algorithm can be used with most commercial FE software. In this paper, we adopted Abaqus [1]. A Tool Command Language (Tcl) [35] script is used to orchestrate the whole process involving the two-solver methodology. Python [48] scripts are used to control the execution of Abaqus and to convert data file formats. The GFEM code is executed using Tcl scripts. The entire process is executed without user intervention.

The computation cost of a non-intrusive/two-solver implementation relative to an intrusive/single-solver one depends on several factors. If the same method and the same solution algorithm are used in both cases, the intrusive one will be slightly more efficient since it does not require writing and reading pseudo-loads and pseudo-solutions to and from data files. The two-solver algorithm presented in this paper is based on static condensation of enrichment degrees of freedom while intrusive implementations of the G/XFEM in Abaqus are typically done through user element libraries. A comparison between the computational cost of this static condensation algorithm and that of solving the global problem with cracks discretized with the G/XFEM is presented in [31]. It was found in that study that if a single crack configuration is considered, the computational cost of the two approaches is close. However, when more than one configuration is considered, the static condensation algorithm leads to considerable computational savings since the factorization of the global stiffness matrix of the un-cracked structure can be recycled several times [31]. This is particularly advantageous in the simulation of crack propagation as shown in [47].

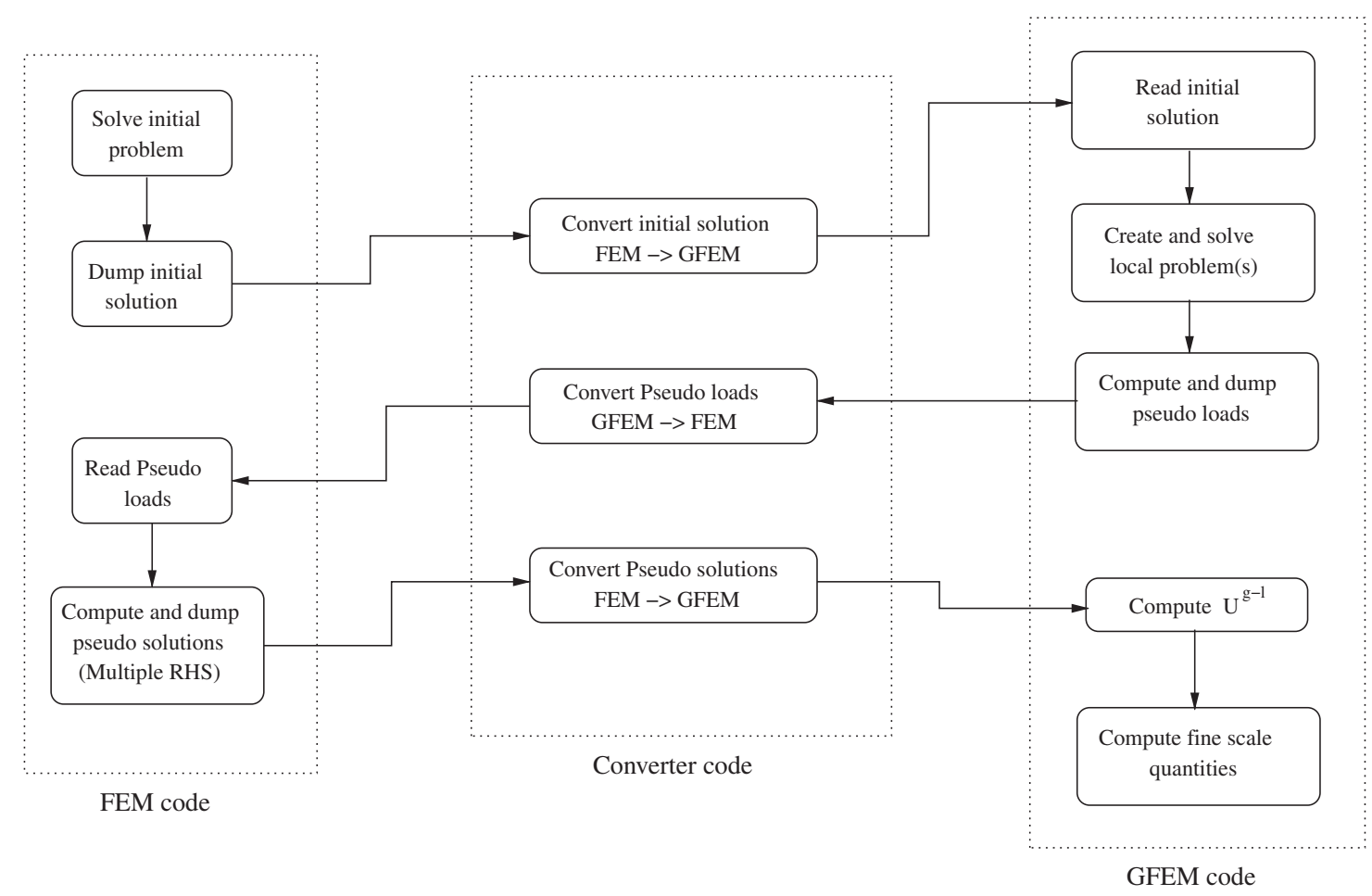

Fig. 4. Flowchart of two-solver algorithm. 


\section{Elements types in global and local discretizations}

The proposed two-solver methodology is based on the GFEM ${ }^{\mathrm{gl}}$ and as such it allows one to use different element types in global and local discretizations. Global elements used in Abaqus provide the partition of unity for the GFEM $^{\text {gl }}$ while local solutions computed by the GFEM software provide enrichment functions. The main requirement on the local meshes is that they are nested in the global one in order to facilitate the numerical integration of matrices (18) and (19) (see also Section 3.5 of [31]). It is even conceivable to use a different numerical method to solve the local problem.

In this paper, Abaqus global meshes use classical linear (TET4) or quadratic (TET10) elements. These elements provide the partition of unity functions for the GFEM ${ }^{\mathrm{gl}}$. Local meshes defined in the GFEM software use high-order elements with shape functions defined as in (7) where a linear partition of unity (TET4) and polynomial enrichment functions are used. Thus, when TET10 elements are used in the global problem, they are first converted to TET4 elements before using them in the construction of local meshes.

Duarte et al. [14] have shown that quadratic GFEM elements with shape functions (7) are more computationally efficient than classical TET10 elements. These element types are also studied in [60,61]. However, since in Abaqus we are limited to classical finite elements, we adopt TET4 or TET10 elements for all global meshes. The performance of these two types of global meshes in the context of the proposed two-solver GFEM $^{\mathrm{gl}}$ is investigated in Section 7.

\section{Numerical examples}

We analyze several examples to verify the accuracy and demonstrate the versatility of the proposed two-solver methodology with $\mathrm{GFEM}^{\mathrm{gl}}$. The solutions of three-dimensional fracture mechanics problems are compared with those available in the literature and with solutions provided by the $h p$-GFEM presented in [31,44,45]. Abaqus [1] is used as the FEA software and ISET (GFEM research code) is used as the GFEM software.

In all examples, coarse, uncracked, global meshes are used. A single local problem is defined for each crack in the domain. Local problems are $h p$-adapted and enriched with both Heaviside and Westergaard singular functions $[44,45]$. The accuracy of GFEM $^{\mathrm{gl}}$ solutions are evaluated in terms of the strain energy norm and stress intensity factor extracted using the Cut-off Function Method (CFM) [43,58]. In order to quantify the error of the stress intensity factors (SIFs) extracted along a crack front, we use a normalized discrete $L^{2}$-norm of the difference between the computed SIF and the reference solution defined by

$$
e^{r}=\frac{\left\|e_{i}\right\|_{L^{2}}}{\left\|\widehat{K}_{i}\right\|_{L^{2}}}=\frac{\sqrt{\sum_{j=1}^{N_{\text {ext }}}\left(K_{i}^{j}-\widehat{K}_{i}^{j}\right)^{2}}}{\sqrt{\sum_{j=1}^{N_{\text {ext }}}\left(\widehat{K}_{i}^{j}\right)^{2}}}
$$

where $N_{\text {ext }}$ is the number of extraction points along the crack front, $\widehat{K}_{i}^{j}$ and $K_{i}^{j}$ are the reference and computed stress intensity factor values for mode $i$ at the crack front point $j$, respectively.

\subsection{Edge crack}

As a first example, a rectangular bar with a through-the-thickness crack is considered. The geometry of the domain and boundary conditions for the problem are illustrated in Fig. 5a. The following geometrical and material parameters are adopted: In-plane dimensions $b=2 h=4.0$; domain thickness $t=1.0$; crack size $a=1.0$; Young's modulus $E=200,000$, Poisson's ratio $v=0.30$. The domain is loaded by a unity tensile traction $T$ (cf. Fig. $5 \mathrm{a}$ ).

The objectives of this example are to evaluate the effects of the size of the local problem domain on the enriched global problem and to investigate the performance of global Abaqus discretizations with TET4 and TET10 elements.

The reference values for Mode I stress intensity factor at the center of the crack front, $\widehat{K}_{I}$, and strain energy, $\widehat{U}$, are computed using an $h p$-GFEM discretization with 405,324 degrees of freedom. In this study, we adopt $\widehat{K}_{I}=3.0796$ and $\widehat{U}=5.55154 \times 10^{-5}$.

The global domain is discretized using a uniform mesh having $6 *(5 \times 5 \times 2)$ tetrahedral elements as shown in Fig. $5 \mathrm{~b}$. This mesh was generated by first creating a $(5 \times 5 \times 2)$ structured mesh of hexahedral elements and then replacing each element by six tetrahedral elements. Linear TET4 or quadratic TET10 elements are used in the global problem solved by Abaqus. The $h p$-GFEM method used to solve local problems adopts linear TET4 elements as a partition of unity, regardless of the element type used in the global problem as discussed in Section 6. Cubic polynomial GFEM shape functions are used in local problems. They are built using quadratic enrichment functions as described in Section 3.

Two local problem domain sizes are used in the numerical experiments. They are defined as follows: Let $\mathscr{I}_{S}^{\text {nlay }=1}$ denote the indices of all clouds $\omega_{\beta}$ from the global mesh that intersect the crack surface $S$. These are also called seed nodes. Local domain $\Omega_{L}^{\text {nlay }=1}$ containing one layer of elements around crack surface $S$ is given by

$$
\Omega_{L}^{\text {nlay }=1}=\bigcup_{\beta \in Y_{S}^{\text {nlay }=1}} \omega_{\beta}
$$




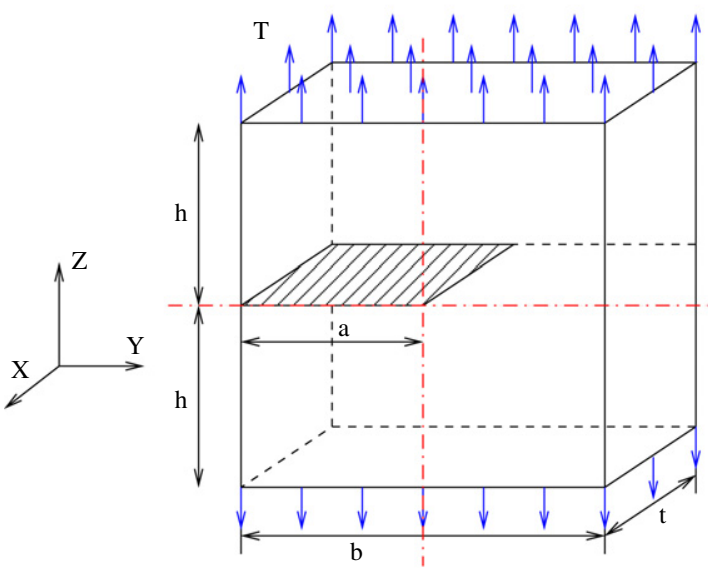

(a) Domain with an edge crack and loaded by tension $T$.

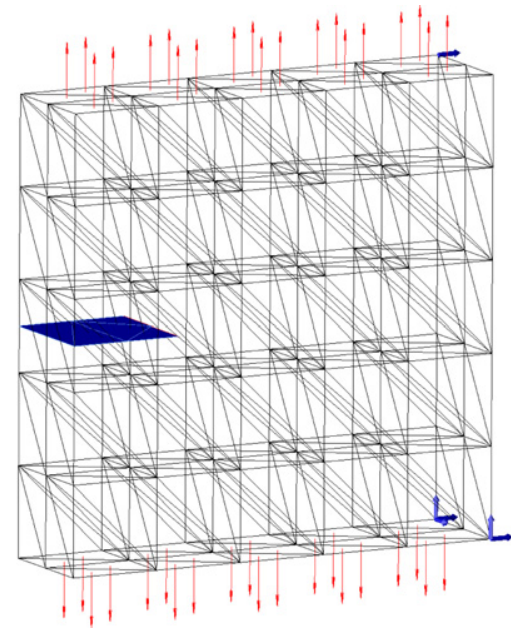

(b) Global mesh used in Abaqus.

The crack is only shown for illustration.

It is not discretized in the global domain.

Fig. 5. Geometry and discretization of the problem domain for the through-the-thickness edge crack problem.

where the cloud $\omega_{\beta}$ is the union of (copy of) global elements sharing vertex node $\beta, \beta \in \mathscr{I}_{S}^{\text {nlay=1}}$. Larger local domains containing additional layers of elements around the crack surface are defined analogously:

$$
\Omega_{L}^{\text {nlay }=\mathrm{m}}=\bigcup_{\beta \in \mathcal{S}_{S}^{\text {nlay }=\mathrm{m}}} \omega_{\beta}
$$

where $\mathscr{I}_{S}^{\text {nlay=m }}, m \geqslant 2$, denotes the indices of all clouds from the global mesh that intersect the local domain $\Omega_{L}^{\text {nlay=m-1 }}$. Figs. 6 and 7 illustrate local domains $\Omega_{L}^{\text {nlay }=1}$ and $\Omega_{L}^{\text {nlay }=2}$, respectively, used in this problem.

The local elements intersecting the crack front are bisected until an acceptable level of mesh refinement is achieved (cf. Figs. 6 and 7a). The ratio of element size to crack length $\left(L_{e} / a\right)$ for elements along the crack front is 0.025 . The longest edge of an element is used as a measure of its size. Spring boundary conditions provided by the initial global solution computed by Abaqus are applied to the local problems. The spring stiffness is calculated using Eq. (12). The solution of the local problem is used as enrichment functions for the enriched global problem as described in Section 4.3.

The various cases analyzed are presented in Table 1. Local problem domains (column 2) are defined in (25) and (26). Enrichment zones used in the global problem (column 3) are defined by the indices of global nodes enriched with

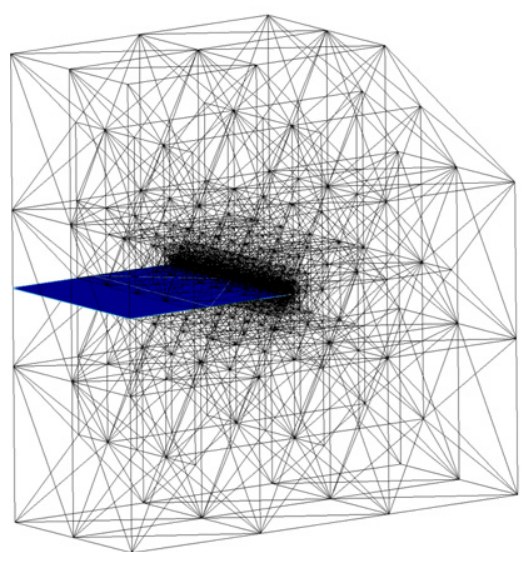

(a) $h p$-adapted local discretization.

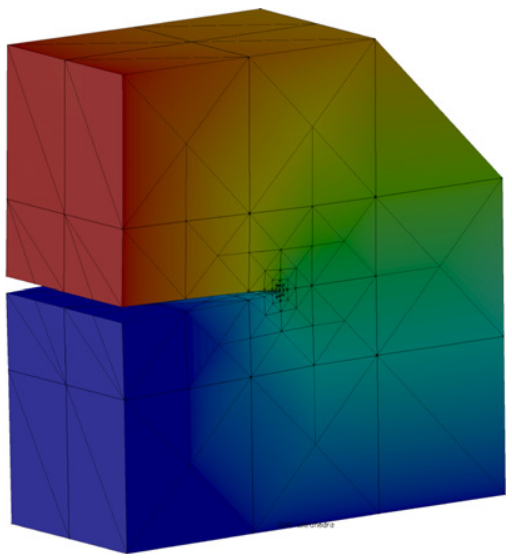

(b) Contour of solution component in y-direction for local problem on $\Omega_{L}^{\text {nlay=1}}$.

Fig. 6. Local domain $\Omega_{L}^{\text {nlay=1}}$ used to compute a global-local enrichment function. 


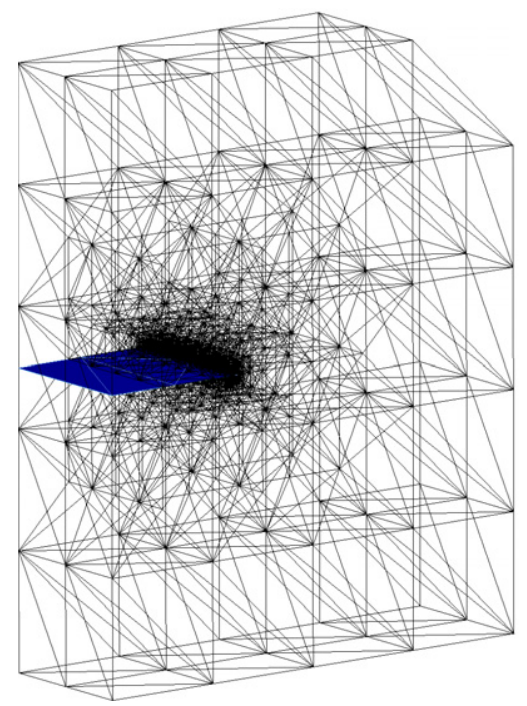

(a) $h p$-adapted local discretization.

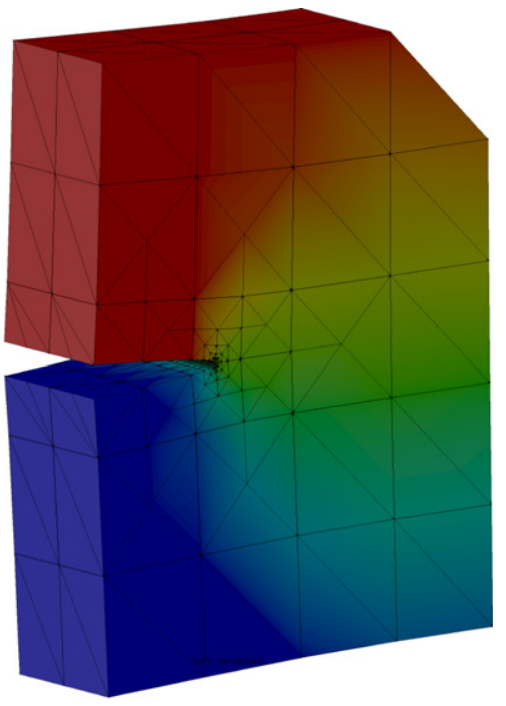

(b) Contour of solution component in y-direction for local problem on $\Omega_{L}^{\text {nlay=2}}$.

Fig. 7. Local domain $\Omega_{L}^{\text {nlay=2}}$ used to compute a global-local enrichment function.

Table 1

Parameters for discretizations used with edge crack problem.

\begin{tabular}{|c|c|c|c|c|c|}
\hline Case & Local dom. & Enrich. zone & Global elem. type & $p$-order & Num. enrich. \\
\hline 1 & $\Omega_{L}^{\text {nlay }=1}$ & $\mathscr{I}_{S}^{\text {nlay }=1}$ & TET4 & 1 & 54 \\
\hline 2 & $\Omega_{L}^{\text {nlay }=2}$ & $\mathscr{I}_{S}^{\text {nlay }=2}$ & TET4 & 1 & 144 \\
\hline 3 & $\Omega_{L}^{\text {nlay }=1}$ & $\mathscr{I}_{S}^{\text {nlay }=1}$ & TET4 & 2 & 1026 \\
\hline 4 & $\Omega_{L}^{\text {nlay }=2}$ & $\mathscr{I}_{S}^{\text {nlay }=2}$ & TET4 & 2 & 1116 \\
\hline 5 & $\Omega_{L}^{\text {nlay }=1}$ & $\mathscr{I}_{S}^{\text {nlay }=1}$ & TET10 & 2 & 225 \\
\hline 6 & $\Omega_{L}^{\text {nlay }=2}$ & $\mathscr{I}_{S}^{\text {nlay }=2}$ & TET10 & 2 & 735 \\
\hline
\end{tabular}

global-local functions. "Global elem. type" denotes the type of element used in the global problem domain. The initial global problem discretized with TET4 elements has 324 degrees of freedom (dofs) while the discretization with TET10 elements has 1815 dofs. "p-order" denotes the polynomial order of the shape functions used in the enriched global approximation. Discretizations 3 and 4 use, in addition to global-local functions, linear polynomial enrichments which lead to quadratic GFEM shape functions. The polynomial enrichments are used in all global nodes. "Num. enrich." denotes the number of globallocal, and polynomial enrichment functions in the case of discretizations 3 and 4, added to the global problem.

Table 2 presents the results for stress intensity factor $K_{I}$ and strain energy $U$ for the discretizations defined in Table 1 . The relative error of stress intensity factor, $e^{r}\left(K_{I}\right)$, is computed at the center of the crack front, i.e., at $z=t / 2$, using

$$
e^{r}\left(K_{I}\right)=\frac{\left|K_{I}-\widehat{K}_{I}\right|}{\widehat{K}_{I}} 100
$$

where $\widehat{K}_{I}=3.0796$ is the reference value. The relative error in strain energy, $e^{r}(U)$, is computed using

$$
e^{r}(U)=\frac{|U-\widehat{U}|}{\widehat{U}} 100
$$

where $\widehat{U}=5.55154 \times 10^{-5}$ is the reference value.

The relative error of stress intensity factor extracted from local solutions computed on the domains shown in Figs. 6 and 7 are $57.56 \%$ and $4.38 \%$, respectively.

The data in Table 2 shows that Abaqus discretizations with linear shape functions lead to large errors in both strain energy and stress intensity factor. Increasing the local domain size and the number of nodes enriched with global-local functions (Case 2) reduce the error but not to an acceptable level. If polynomial enrichments are used (Cases 3 and 4 ), there is a dramatic reduction in the error of strain energy and stress intensity factor even when the small local problem domain, 
Table 2

Stress intensity factor $K_{I}$ and strain energy for edge crack problem. Enriched global problem results.

\begin{tabular}{lllll}
\hline Case ID & $K_{I}$ & $U \times 10^{5}$ & $e^{r}\left(K_{I}\right)(\%)$ & $e^{r}(U)(\%)$ \\
\hline 1 & 2.244 & 5.114 & 27.143 & 7.8729 \\
2 & 2.542 & 5.263 & 17.469 & 5.1999 \\
3 & 3.024 & 5.508 & 1.8167 & 0.7856 \\
4 & 3.085 & 5.544 & 0.1649 & 0.1362 \\
5 & 2.885 & 5.348 & 6.3266 & 3.6659 \\
6 & 3.089 & 5.546 & 0.2960 & 0.1006 \\
\hline
\end{tabular}

$\Omega_{L}^{\text {nlay=1}}$, is used. These discretizations, however, require the exchange of a large number of pseudo-loads and pseudo-solutions between Abaqus and the GFEM code (1026 for Case 3 instead of 54 for Case 1 where no polynomial enrichments are used). Discretizations 5 and 6 use TET10 elements in the global Abaqus mesh and are enriched with global-local functions only. They deliver significantly lower error levels than Cases 1 and 2 while using fewer enrichments than Cases 3 and 4.

The above results show that there are at least three ways to improve the accuracy of the method, namely (i) take higherorder elements in the global mesh, (ii) use polynomial enrichment functions at global nodes (in addition to global-local functions) and (iii) increase the size of the local domain and enrichment zone in the global mesh. Improving the accuracy of the local solution through mesh refinement and/or enrichment also improves the solution of the enriched global problem while keeping the global problem at the same size [13,28].

The effectiveness and cost of strategies (i)-(iii) can be assessed from Tables 1 and 2 . They show that option (ii) leads to a lower error in stress intensity factor than option (i). However it leads to more degrees of freedom in the global problem. Thus, for a two-solver implementation based on static condensation of enrichment degrees of freedom, option (i) is preferred. In the single-solver case, the polynomial enrichments can be used in the initial global problem and thus they do not need to be condensed out. In this case option (ii) is more effective since it leads to a lower error in stress intensity factor than option (i). Increasing the size of the local domains improves the quality of the local solution since it reduces the effect of inexact boundary conditions applied to local problems. An a priori error estimate demonstrating this is presented in [28]. The optimum size of the local domain can only be determined using a posteriori error measures. Our numerical experience shows that in the case of linear elastic fracture problems in 3-D, the local domains should be selected such that the crack front is not close to the local domain boundary. Since we are mainly interested in small cracks and coarse meshes in the global problem, this condition is easily met.

\subsection{Small surface crack}

The second problem considered is a small half-penny surface-breaking crack as illustrated in Fig. 8a. This problem has been analyzed by several researchers $[49,50,62]$ using the finite element method. The curved crack front creates more pronounced 3-D effects than in the previous example. The following geometrical and material parameters are adopted: In-plane dimensions $2 b=2 h=2.0$; domain thickness $t=1.0$; crack radius $r=0.2$; Young's modulus $E=1.0$, Poisson's ratio $v=0.25$. The domain is loaded by a unity tensile traction $T$ as illustrated in Fig. 8a.

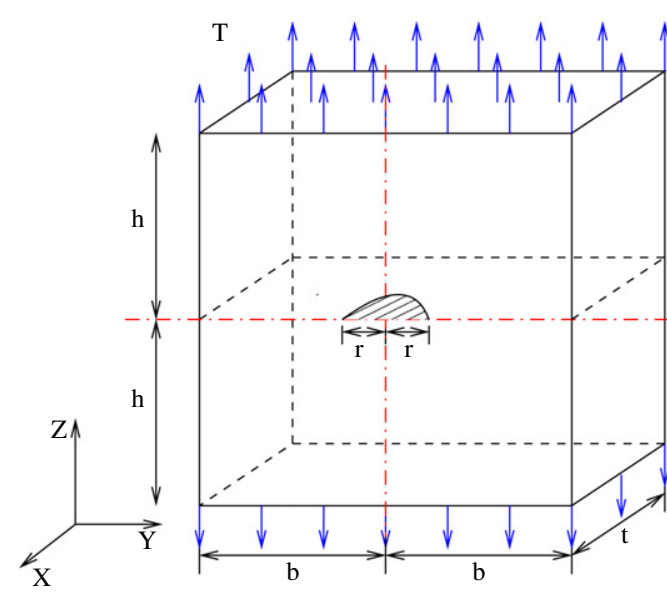

(a) Domain with a small surface crack and loaded by a tension $T$.

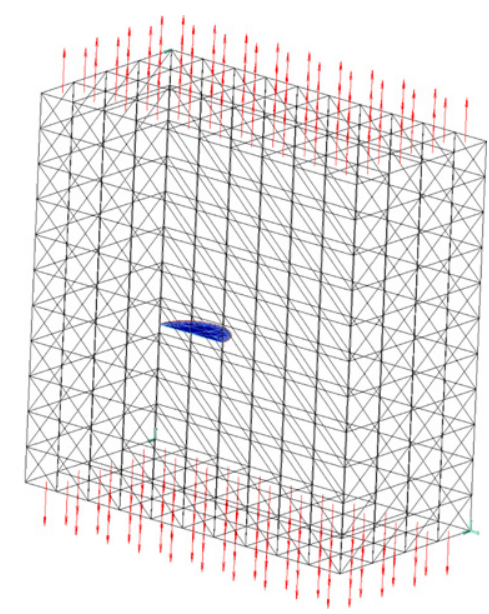

(b) Global mesh used in Abaqus. The crack is not discretized in the global domain.

Fig. 8. Geometry and discretization of the problem domain for the small surface crack problem. 
The global domain is discretized with a uniform coarse mesh of $6 *(10 \times 11 \times 4)$ TET4 or TET10 elements as shown in Fig. 8b. This is a very coarse mesh with element sizes almost equal to the radius of the crack. The initial global problem discretized with TET4 elements has 1980 dofs while in the case of TET10 elements it has 13,041 dofs. These problems are solved by Abaqus.

Two local problem domain sizes, $\Omega_{L}^{\text {nlay=1}}$ and $\Omega_{L}^{\text {nlay=2}}$ are used in the numerical experiments. They are defined as in the previous section. Figs. 9 and 10 show the meshes used in these domains. Cubic polynomial shape functions are used in local problems. Local elements intersecting the crack front are bisected until an acceptable level of mesh refinement is achieved. The ratio of element size to crack radius $\left(L_{e} / r\right)$ along the crack front is 0.0287 . The von Mises stress in the local domains is shown in Figs. 9b and 10b. The GFEM ${ }^{\mathrm{gl}}$ solution is computed following the two-solver methodology described in Section 5.

Quality of Extracted Stress Intensity factors Mode I stress intensity factor, $K_{I}$, extracted along the crack front is normalized using

$$
\bar{K}_{I}=\frac{K_{I}}{T \sqrt{\frac{\pi r}{Q}}}
$$

where $Q$ is equal to 2.464 for a circular crack, $T$ is the tensile stress and $r$ is the radius of the crack. The reference values for $\bar{K}_{I}$ are taken from Walters et al. [62] and are used to compute $e^{r}\left(\bar{K}_{I}\right)$ using Eq. (24). The relative error $e^{r}\left(\bar{K}_{I}\right)$ of stress intensity factors extracted from local solutions, $\boldsymbol{u}_{L}$, computed on the domains shown in Figs. 9 and 10 are $8.718 \%$ and $4.709 \%$, respectively. This is the error of a standard global-local analysis (GL-FEM).

Table 3 lists the parameters for the various discretizations considered and the relative error $e^{r}\left(\bar{K}_{I}\right)$ in the enriched global problem. It can be observed from the results that the error in $\bar{K}_{I}$ is substantial for Abaqus discretizations with linear shape

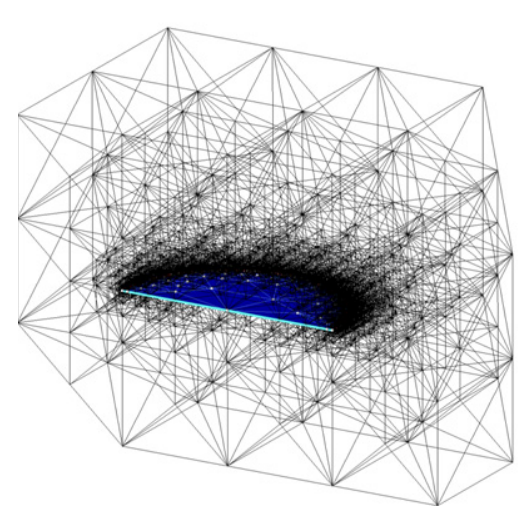

(a) $h p$-adapted local problem.

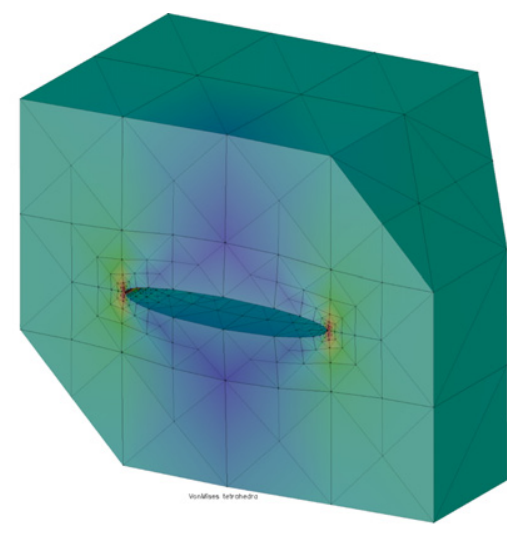

(b) Contour plot of von Mises stress for local problem on $\Omega_{L}^{\text {nlay=1}}$.

Fig. 9. Local problem domain $\Omega_{L}^{\text {nlay=1}}$ used to compute global-local enrichment functions.

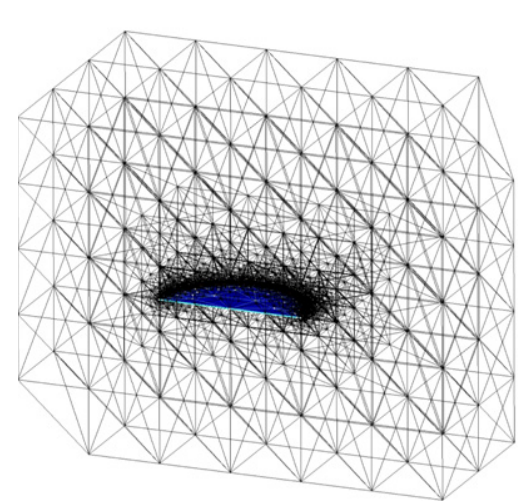

(a) $h p$-adapted local problem.

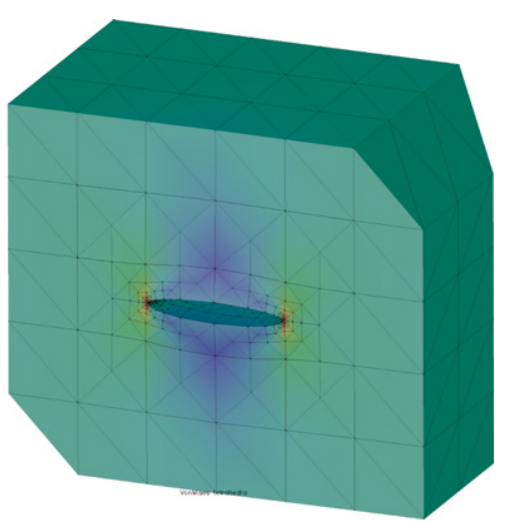

(b) Contour plot of von Mises stress for local problem on $\Omega_{L}^{\text {nlay=2}}$.

Fig. 10. Local problem domain $\Omega_{L}^{\text {nlay=2}}$ used to compute global-local enrichment functions. 
Table 3

Parameters and error in $\bar{K}_{I}$ for enriched global discretizations used with the surface crack problem.

\begin{tabular}{|c|c|c|c|c|c|c|}
\hline Case & Local dom. & Enrich. zone & Global elem. type & p-Order & Num. enrich. & $e^{r}\left(\bar{K}_{I}\right)(\%)$ \\
\hline 1 & $\Omega_{L}^{\text {nlay }=1}$ & $\mathscr{I}_{S}^{\text {nlay }=1}$ & TET4 & 1 & 36 & 17.7788 \\
\hline 2 & $\Omega_{L}^{L}$ nlay $=2$ & $\mathscr{I}_{S}^{\text {nlay }=1}$ & TET4 & 1 & 36 & 17.3540 \\
\hline 3 & $\Omega_{L}^{\text {nlay }=2}$ & $\mathscr{I}_{S}^{\text {nlay }=2}$ & TET4 & 1 & 180 & 14.8951 \\
\hline 4 & $\Omega_{L}^{\mathrm{nlay}=1}$ & $\mathscr{I}_{S}^{\text {nlay }=1}$ & TET4 & 2 & 5976 & 1.3355 \\
\hline 5 & $\Omega_{L}^{\text {nlay }=2}$ & $\mathscr{I}_{S}^{\text {nlay }=1}$ & TET4 & 2 & 5976 & 1.2214 \\
\hline 6 & $\Omega_{L}^{\text {nlay }=2}$ & $\mathscr{I}_{S}^{\text {nlay }=2}$ & TET4 & 2 & 6120 & 0.5287 \\
\hline 7 & $\Omega_{L}^{\mathrm{nlay}=1}$ & $\mathscr{I}_{S}^{\text {nlay }=1}$ & TET10 & 2 & 135 & 3.0459 \\
\hline 8 & $\Omega_{L}^{\text {nlay }=2}$ & $\mathscr{I}_{S}^{\text {nlay=1 }}$ & TET10 & 2 & 135 & 2.9913 \\
\hline 9 & $\Omega_{L}^{\text {nlay }=2}$ & $\mathscr{I}_{S}^{\text {nlay }=2}$ & TET10 & 2 & 945 & 0.5941 \\
\hline
\end{tabular}

functions (Cases 1,2 and 3). Increasing the local domain size and the number of nodes enriched with global-local functions reduce the error but not to an acceptable level. It can be noted from Cases 4-6 that the quality of $\bar{K}_{I}$ is greatly improved when linear polynomial enrichment functions are also used along with global-local enrichment functions. However, the number of enrichment functions increase significantly. A high level of accuracy in $\bar{K}_{I}$ can also be achieved by using TET10 elements in the global Abaqus mesh (Cases 7-9). The error in $\bar{K}_{I}$ is comparable with those from Cases 4-6 while using significantly fewer enrichments.

Fig. 11a and b present the normalized $\bar{K}_{I}$ extracted along the crack front using discretizations $1-3$ and $4-6$, respectively. The position along the crack front is defined by the polar angle $\phi$ where $\phi=0$ and $\phi=\pi / 2$ correspond to a vertex of the crack front and the center of the crack front, respectively. It can be noted from Fig. $11 \mathrm{~b}$ that the quality of $\bar{K}_{I}$ is greatly improved when linear polynomial enrichment functions are also used along with global-local enrichments. Fig. 12 shows the results for cases with TET10 elements in the global Abaqus mesh. It can be observed that enriching two layers of global nodes around the crack leads to a very good matching with the reference solution along the entire crack front. This demonstrates the versatility of the proposed two-solver methodology where different types of elements can be used in global and local problems and convergence studies can be performed by changing the size of the enrichment zone while not requiring any modifications to the global mesh used by Abaqus.

\subsection{Interacting cracks}

In this section the problem of two interacting cracks in a strip is analyzed. The problem is illustrated in Fig. 13. The general plane elastic problem of an infinite strip containing multiple cracks perpendicular to its boundary was analyzed by Civelek and Erdogan [9]. They showed that for the configuration shown in Fig. 13, the interaction between the cracks produces a nonzero mode-two stress intensity factor, $K_{I I}$. This leads to the propagation of the cracks away from each other. This effect becomes more significant as the distance between the cracks decreases [9]. This problem was also analyzed by Kim et al. [30]. In this section, we investigate how well the two-solver GFEM $^{\text {gl }}$ methodology and the classical GL-FEM can capture the interactions between the cracks as the distance between them decreases.

The material and geometrical parameters adopted are as follows: Young's modulus $E=200,000$; Poisson's ratio $v=0.0$; inplane dimensions $H=10.0, V=198.0$; domain thickness $t=1.0$; cracks of size $2 l=4.0$; magnitude of vertical traction $t_{y}=100.0$. Since the vertical dimension is about twenty times larger than the horizontal dimension, we can assume that the solution on this finite domain is close to the case of an infinite strip. Poisson's ratio is set to zero in order to minimize three-dimensional effects. This enables us to use Civelek and Erdogan's 2-D solution presented in [9] as a reference. The global domain is discretized with a uniform coarse mesh of $6 *(10 \times 220 \times 1)$ TET10 elements. This mesh is quite coarse and has only one layer of elements in the out-of-plane direction. The initial global problem has 83,349 dofs and is solved by Abaqus.

The SIFs are extracted using the cut-off function method $[42,43,58]$ and normalized as in [9] using

$$
k_{I(I I)}=\frac{K_{I(I I)}}{t_{y} \sqrt{\pi l}}
$$

where $k_{I(I I)}$ denotes the normalized mode I (II) SIF, $K_{I(I I)}$ denotes the computed mode I (II) SIF, $t_{y}$ is the traction applied at $y= \pm V / 2$, and 21 is the crack length. The SIFs are extracted at the center of the crack front.

Two local problems are created, one for each crack as illustrated in Fig. 14. Each local domain is defined using two layers of global elements around the crack, i.e., local domain size is $\Omega_{L}^{\text {nlay }=2}$. Local meshes with cubic shape functions are refined in the neighborhood of crack fronts as shown in Fig. 14. For the GL-FEM, the stress intensity factors are extracted from local solutions. Since no cracks are discretized in the initial global problem and the local problems are solved independently, they cannot capture the interactions between cracks. In the GFEM ${ }^{\text {gl }}$, each local solution is used to enrich 273 nodes in the global 


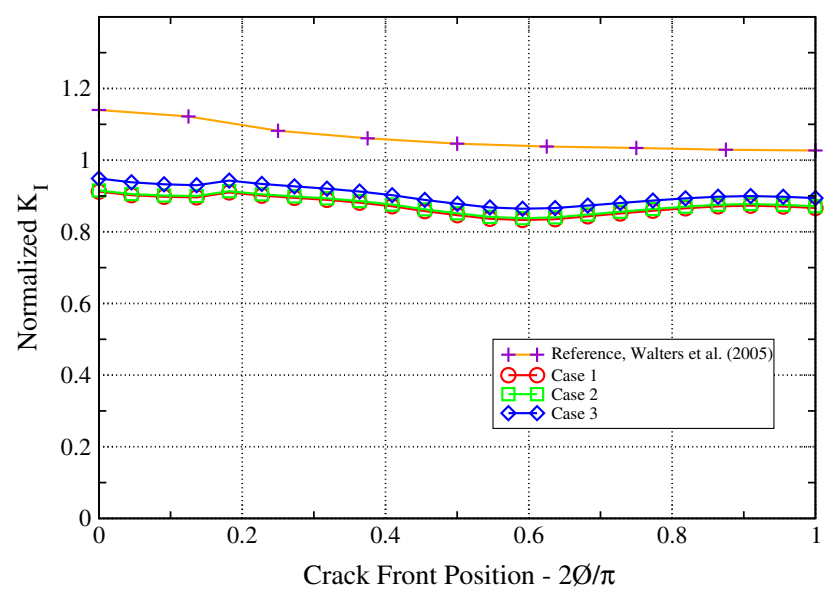

(a)

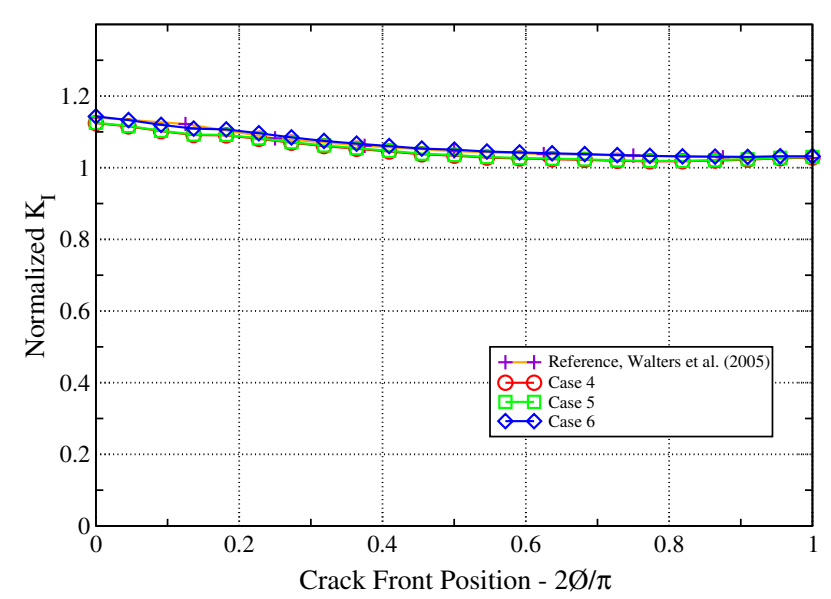

(b)

Fig. 11. Mode I stress intensity factor $K_{I}$ for surface crack problem solved with TET4 elements in the global problem. (a) Only global-local enrichments are used. (b) Linear polynomial enrichment functions are used along with global-local enrichment functions.

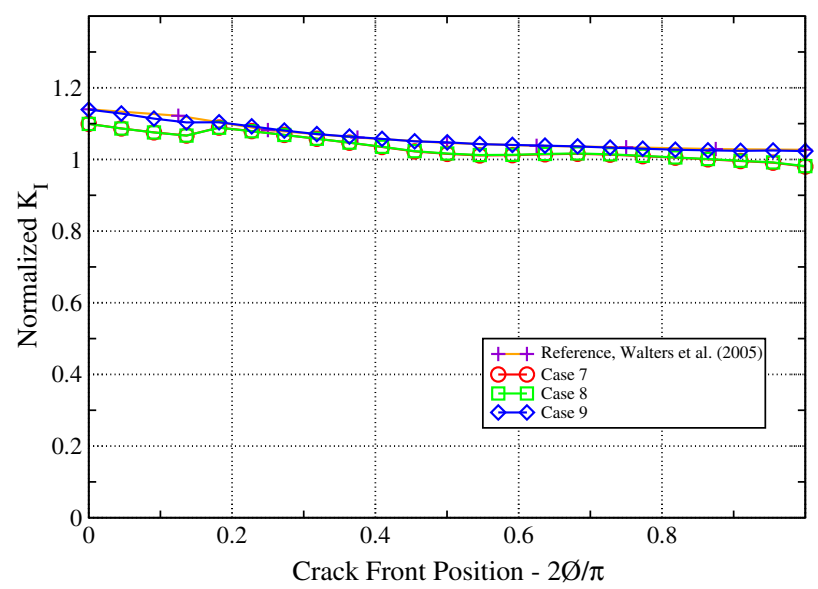

Fig. 12. Mode I stress intensity factor $K_{I}$ for surface crack problem solved with TET10 elements in the global Abaqus mesh. 


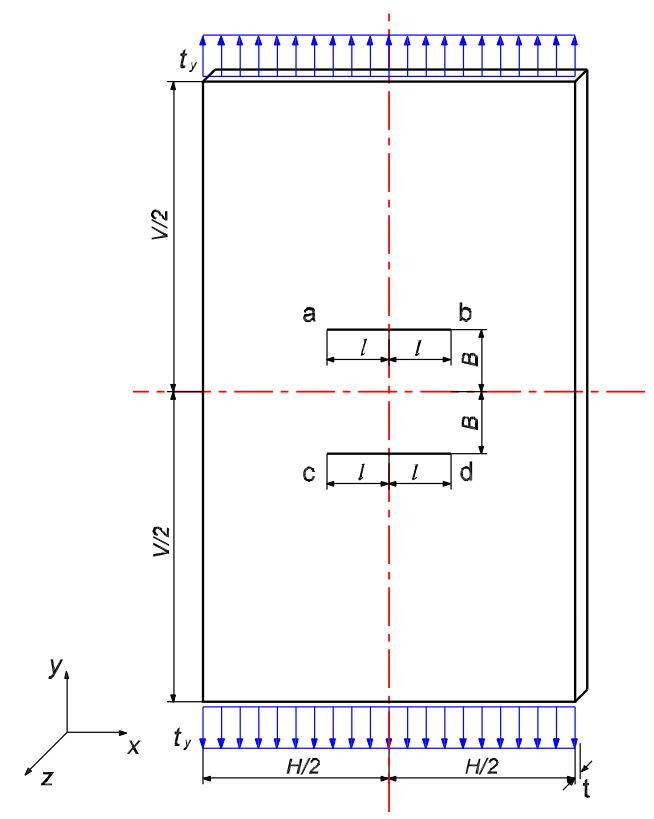

Fig. 13. Description of a problem with two interacting cracks.

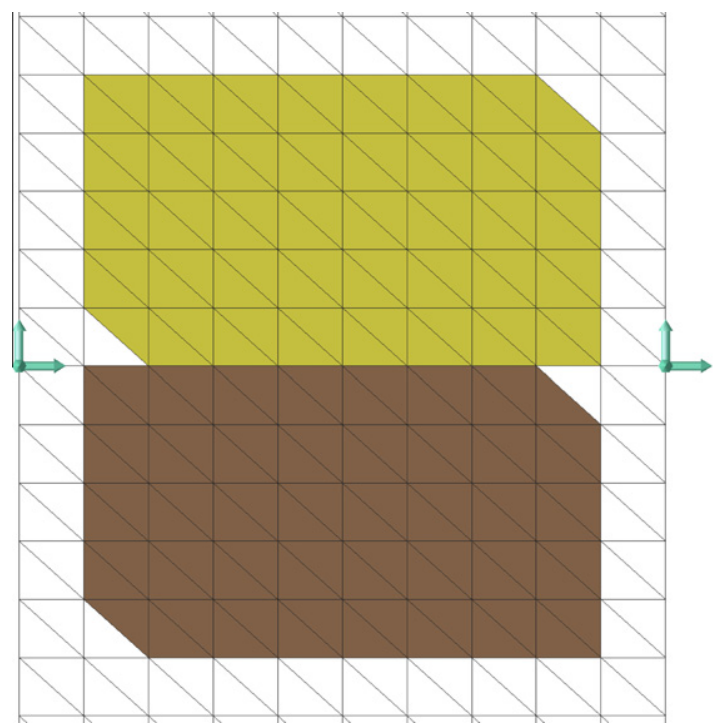

Local Problem for crack 1

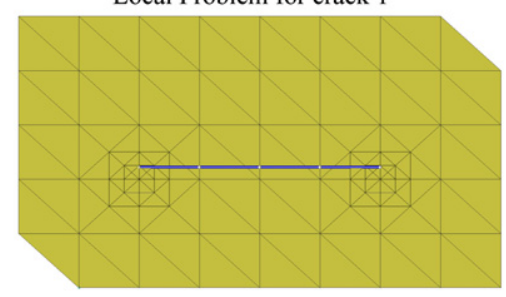

Local Problem for crack 2

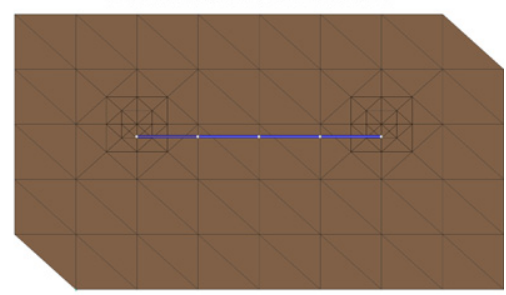

Fig. 14. Shaded areas represent the local problem domain for $B / H=0.2$. $h p$-GFEM discretizations used to solve the local problems are also shown in the figure.

mesh. This leads to a total of 1638 enrichments in the enriched global problem which is about $2 \%$ of the number of dofs of the initial global problem.

Table 4 lists the computed (normalized) mode I and II SIFs ( $k_{I}$ and $\left.k_{I I}\right)$ extracted from GL-FEM and two-solver GFEM ${ }^{\text {gl }}$ solu- $^{-}$ tions. The reference values from Civelek and Erdogan [9] are also listed. Fig. 15 plots the data from the table. The two-solver GFEM $^{\text {gl }}$ can capture very well the interactions between the cracks while the GL-FEM results are quite poor. For the case where maximum interaction between the cracks is present, i.e., for $B / H=0.2$, the relative error of $k_{I}$ extracted from the GFEM $^{\text {gl }}$ solution is $0.83 \%$, whereas the error is $31.35 \%$ in the case of the GL-FEM. A similar trend can be seen for other values of $\mathrm{B} / \mathrm{H}$. Mode II SIFs computed by both methods are not as accurate as the case of mode I SIFs. This can be attributed to the fact that for this problem $k_{I I}$ is much smaller than $k_{I}$ and hence it cannot be computed as accurately as $k_{I}$ using either method unless finer meshes are used. The boundary conditions used at local problems are independent of $B / H$ since they come from a global problem without cracks. This explains why the value of $k_{I}$ extracted from the GL-FEM solution is independent of B/H. 
Table 4

Mode I and II stress intensity factors for the problem shown in Fig. 13. Cracks are not discretized in the global problem solved by Abaqus. Abbreviations nDOFs, IG, L and EG in the table represent the number of degrees of freedom, initial global, local and enriched global problems, respectively.

\begin{tabular}{|c|c|c|c|c|c|c|c|c|c|}
\hline \multirow[t]{2}{*}{$B / H$} & \multirow[t]{2}{*}{ nDOFs (IG) } & \multirow[t]{2}{*}{ nDOFs (L) } & \multirow[t]{2}{*}{ nDOFs (EG) } & \multicolumn{3}{|c|}{ Normalized Mode I SIF } & \multicolumn{3}{|c|}{ Normalized Mode II SIF } \\
\hline & & & & GL-FEM & GFEM $^{\mathrm{gl}}$ & Ref. & GL-FEM & GFEM $^{\mathrm{gl}}$ & Ref. \\
\hline 0.2 & 83,349 & 9120 & 84,987 & 0.6693 & 0.9668 & 0.9749 & -0.0083 & -0.0436 & -0.0656 \\
\hline 0.3 & 83,349 & 9840 & 84,987 & 0.6693 & 1.0402 & 1.0437 & -0.0062 & -0.0211 & -0.0330 \\
\hline 0.4 & 83,349 & 9840 & 84,987 & 0.6693 & 1.0818 & 1.0839 & -0.0008 & -0.0095 & -0.0155 \\
\hline 1.0 & 83,349 & 9120 & 84,987 & 0.6693 & 1.0904 & 1.1096 & & & \\
\hline
\end{tabular}

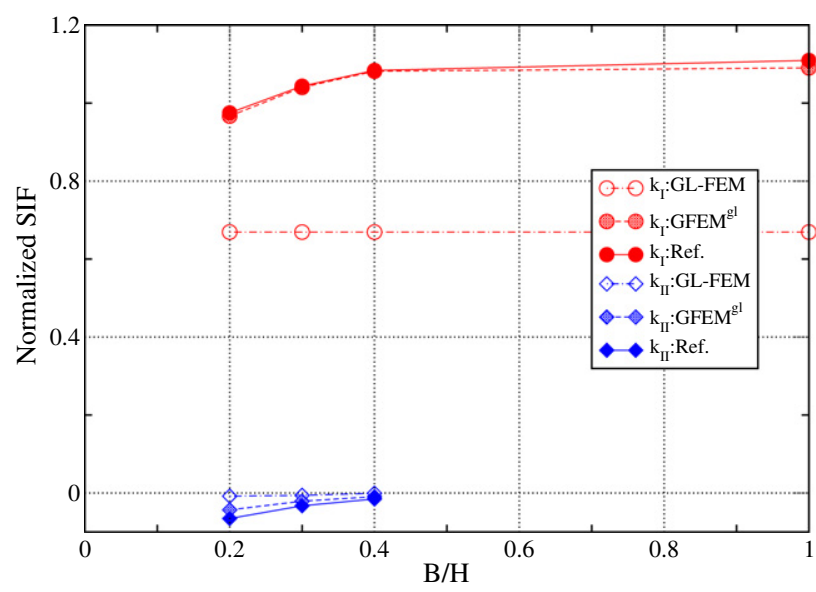

Fig. 15. Analysis of interacting cracks in a strip. The global problem is solved with TET10 elements in Abaqus.
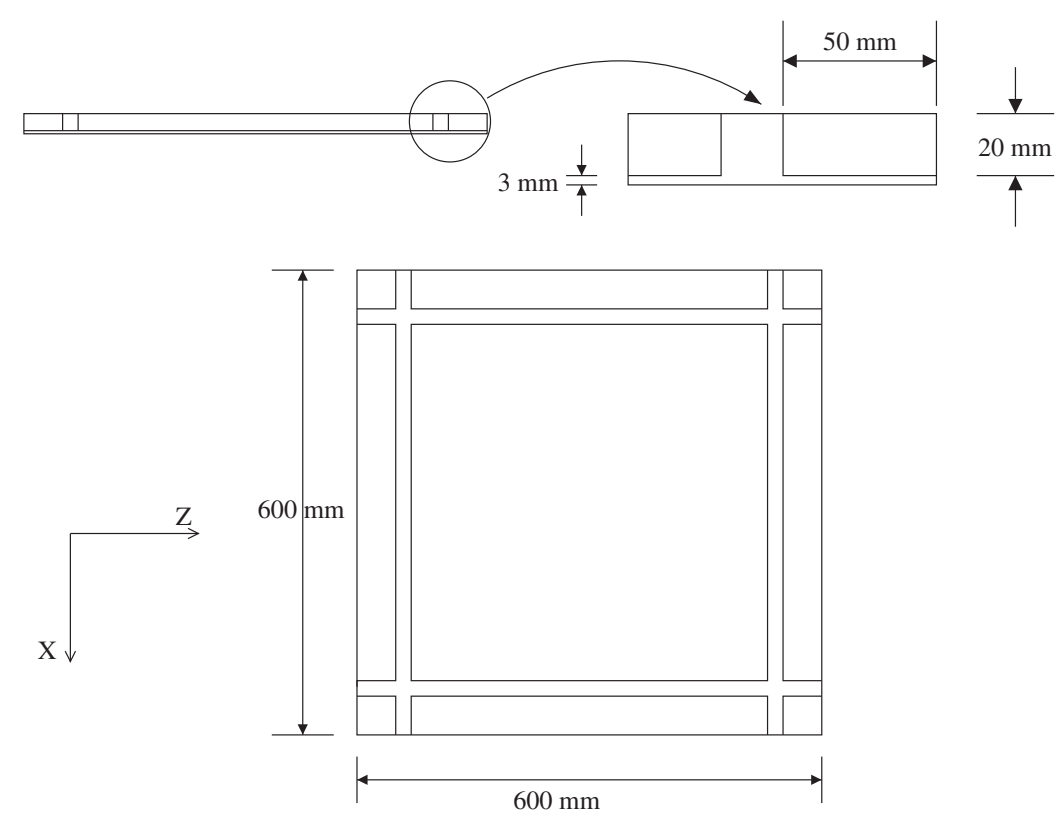

Fig. 16. Geometry of the plane stiffened panel.

In contrast, the $\mathrm{GFEM}^{\mathrm{gl}}$ solution accounts for the crack interactions. The cracks are discretized in the enriched global problem though the global-local enrichments and thus their interactions are properly captured.

The interactions between the cracks increase as $B / H$ decreases. This, as shown above, cannot be captured by the GL-FEM. In the case of the $\mathrm{GFEM}^{\mathrm{gl}}$ the interactions can be captured but if $B / H$ is very small, the local domains will overlap. This poses 


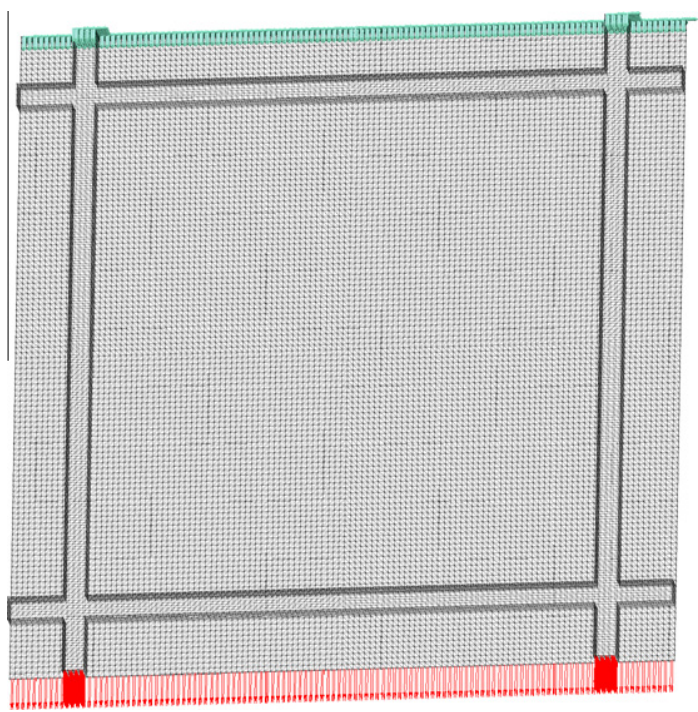

Fig. 17. Boundary conditions and discretization of the global problem solved by Abaqus.

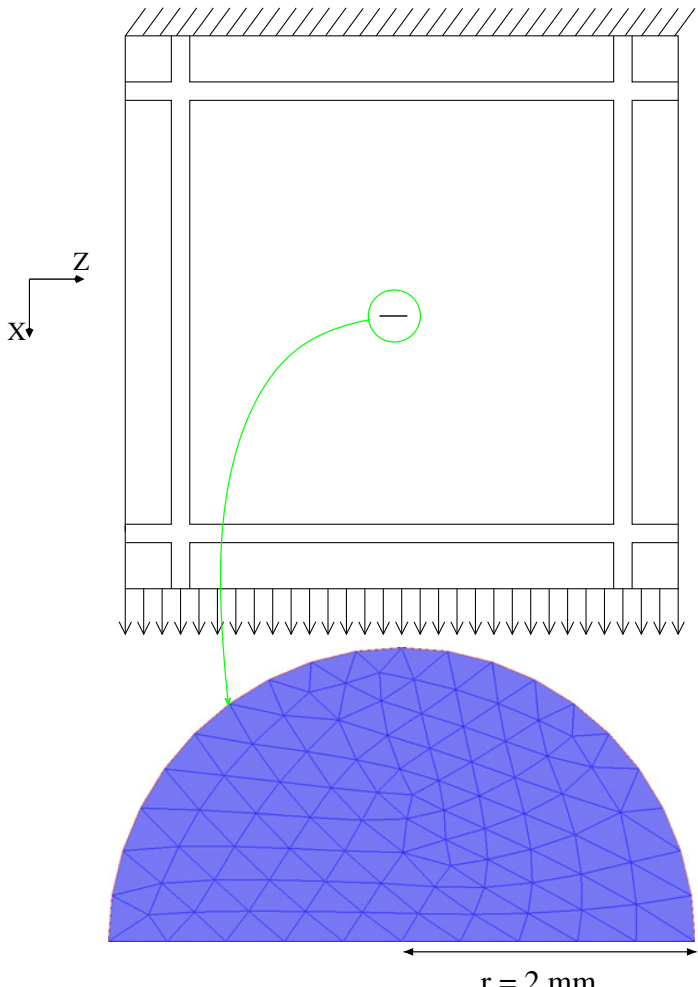

Fig. 18. Description of the stiffened panel subjected to uni-directional tension with a half-penny surface crack near the center of the panel. The panel is fixed at the top edge and subjected to uniform tractions in the positive $x$-direction at the bottom edge.

no difficulty to compute global-local enrichments since the local problems are solved independently. However, if a finite element from the global problem is enriched with solutions from different local problems, their meshes should be the same over this global element. This allows the numerical integration of the stiffness matrix of this element using either one of the local meshes. Further details about his issue and a simple algorithm to guarantee compatibility of local meshes are presented in [33]. Another option to handle the case of small $B / H$ is to defined a single local problem containing both cracks. 


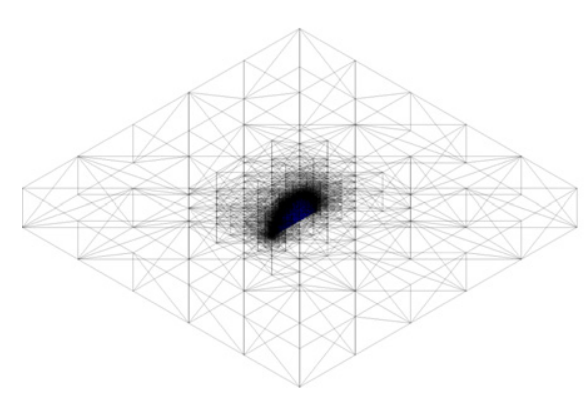

(a) $h p$-adapted local problem for half-penny surface crack

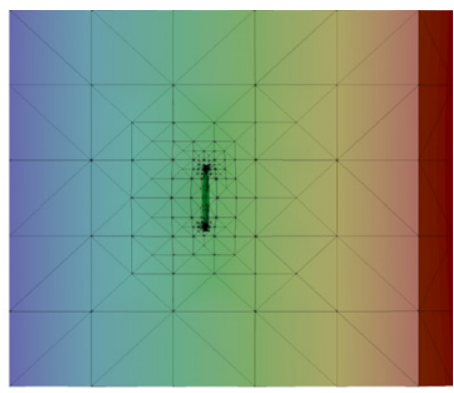

(b) Contour of solution for local problem showing crack opening.

Fig. 19. Local problem domain $\Omega_{L}^{\text {nlay=2 }}$ used to compute global-local enrichment functions for a half-penny surface crack.

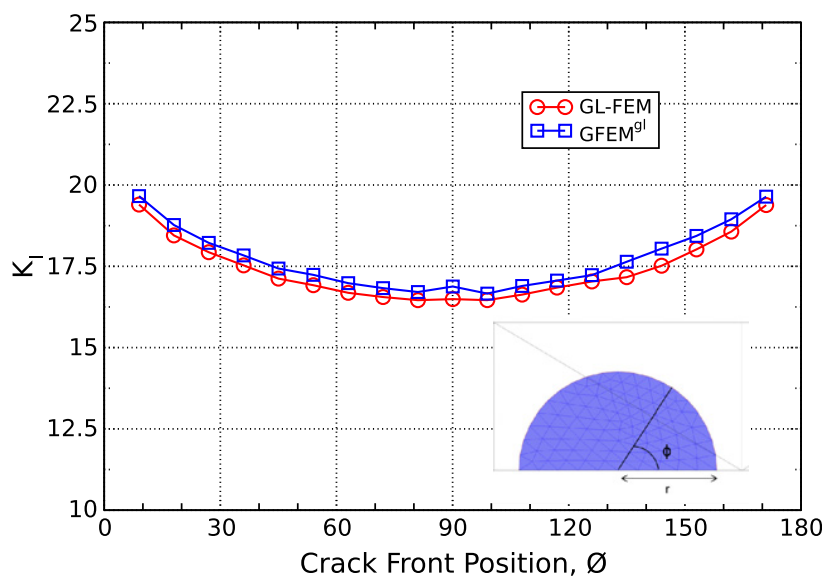

Fig. 20. Mode I stress intensity factor $K_{I}$ for stiffened panel solved with TET10 elements in the global problem. The insert in thefigure shows the crack surface and TET10 elements from the global mesh.

\subsection{Stiffened panel}

The problem considered in this section is motivated by case studies in structural integrity assessment of aeronautical structures [63]. The goal of this study is to find the stress intensity factors for a small crack in a plane one-bay stiffened panel subjected to a $10 \mathrm{MPa}$ uni-directional tension. The geometry and material properties are adopted from [63]. The panel is a $600 \mathrm{~mm} \times 600 \mathrm{~mm} \times 3 \mathrm{~mm}$ square sheet stiffened by four beams with a $20 \mathrm{~mm} \times 20 \mathrm{~mm}$ square section (cf. Fig. 16). The panel is made of aluminum: Young's modulus $E=72,000 \mathrm{MPa}$ and Poisson's ratio $v=0.33$. The stiffeners are made of steel: Young's modulus $E=210,000 \mathrm{MPa}$ and Poisson's ratio $v=0.3$.

A half-penny surface crack is inserted as illustrated in Fig. 18. The crack radius is $2 \mathrm{~mm}$ which is $1 / 300$ of the panel size. The crack is almost at the center of the panel. The coordinates of the crack ends are $(2,0,-4.5)$ and $(2,0,-0.5)$. The panel is discretized using TET10 elements as shown in the Fig. 17.

A local problem for the crack was created with local domain size $\Omega_{L}^{\text {nlay=2}}$. Fig. 19 shows the mesh and solution for the local problem. Cubic shape functions are used in the local problem. Enrichment zone size of $\mathscr{I}_{S}^{\text {nlay }=2}$ is used in the global problem. This leads to the enrichment of 147 global nodes which is a small number considering that the global mesh has 291,275 nodes. The $h p$-GFEM solution of this problem is not available. To calculate SIF's accurately using $h p$-GFEM, we need to refine the global mesh such that elements around the crack front are small enough. If the global $h p$-GFEM problem adopts the same $L_{e} / a$ ratio as that used in local problem, the ratio of element size to plate dimension will be about $1 / 15,000$. This, together with polynomial enrichments, lead to an ill-conditioned global stiffness matrix and unreliable results.

Fig. 20 shows the stress intensity factor $K_{I}$ extracted from the local problem and the enriched global problem. We can observe that the shape of the SIF curves is quite similar but the curve for the enriched global problem is shifted up.

\section{Conclusions}

In this paper, we proposed an algorithm for the implementation of the Generalized Finite Element Method with global-local enrichment functions $\left(\mathrm{GFEM}^{\mathrm{gl}}\right)$ using a commercial FEA software and a GFEM software. The implementation 
is non-intrusive, i.e., the closed-source commercial software is not modified. It relies on exchanges of pseudo-load and pseudo-solution vectors between two solvers. Virtually all structural solvers can efficiently deal with multiple loads cases, as this is a common situation in practice. The implementation is thus considerably simpler than those based on user element libraries $[25,26,36,53,54,64,65]$, a feature not available in all structural analysis solvers. From the user stand point, the proposed two-solver algorithm is also more convenient than those based on user elements. It can use FE models of un-cracked global problems without any modifications. This is in contrast with approaches based on user element. In this case, prior to each analysis, the user must change element types in portions of the global model. This must be done for each separate analysis case or crack configuration.

The present method can also be applied to the simulation of propagating cracks. In this case, the enriched global solution from propagation step $k$ provides boundary conditions for the local problem at step $k+1$. The solution of this problem is used as global-local enrichments at step $k+1$. One added benefit of solving a crack propagation problem using this algorithm is that the global problem need not be solved from scratch at every propagation step since the stiffness matrix of the uncracked global model can be recycled at every crack propagation step. We plan to explore these ideas in combination with the nonintrusive two-solver algorithm presented in this paper. Extensions to nonlinear problems are also conceivable. The GFEM ${ }^{\mathrm{gl}}$ has been formulated for nonlinear problem in [29,32]. The case of cohesive cracks is currently under investigation by our research group.

The proposed methodology is demonstrated in this paper using Abaqus [1] and the $h p$ adaptive GFEM software ISET. In this case, data transfer between the two softwares is performed by Python scripts and simple data format converters. We present the main algorithm for solving 3-D fracture mechanics problems; the interaction of Python scripts with the FEA and GFEM softwares and the output for post-processing. The versatility and accuracy of the methodology are investigated through several 3-D fracture mechanics examples. Other features of the proposed method and conclusions from the results presented here are listed below.

1. Phenomena involving more than one scale of interest like small cracks in large structures, can be captured in structural scale FEM meshes using existing FEA software. In the examples presented in Section 7, the elements of the global mesh are about the same size as the cracks;

2. The proposed algorithm allows easy transition of multiscale and adaptive mesh refinement/enrichment capabilities of the GFEM $^{\mathrm{gl}}$ to virtually any FEA package. In this paper this is demonstrated with Abaqus. Other FEA softwares can be used as well;

3. The extraction of local problems from global models do not require user intervention. Local meshes are automatically refined and enriched with polynomials as well as functions able to represent cracks that cut elements in the mesh. This removes the need of creating 3-D meshes fitting crack surfaces;

4. The accuracy of quantities of interest is not deteriorated when using the proposed algorithm. The method delivers error levels comparable with standard $h p$-FEM approximations which are known to be very effective for problems exhibiting singularities.

\section{Acknowledgments}

The authors gratefully acknowledge the contributions of the Midwest Structural Sciences Center (MSSC) at the University of Illinois at Urbana-Champaign. The Center is supported by the US Air Force Research Laboratory Air Vehicles Directorate under contract number FA8650-06-2-3620. The support from the US Air Force Research Laboratory Air Vehicles Directorate under contract number USAF-0060-50-0001 is also gratefully acknowledged.

\section{References}

[1] Abaqus. Abaqus user's manual. ABAQUS Inc, Dassault Systèmes. Rhode Island, USA; 2007.

[2] Babuška I, Melenk JM. The partition of unity method. Int J Numer Meth Engng 1997;40:727-58.

[3] Babuška I, Caloz G, Osborn JE. Special finite element methods for a class of second order elliptic problems with rough coefficients. SIAM J Numer Anal 1994;31(4):945-81.

[4] Belytschko T, Black T. Elastic crack growth in finite elements with minimal remeshing. Int J Numer Meth Engng 1999;45:601-20.

[5] Belytschko T, Gracie R, Ventura G. A review of extended/generalized finite element methods for material modeling. Modell Simul Mater Sci Engng 2009;17:24. <http://dx.doi.org/10.1088/0965-0393/17/4/043001>.

[6] Bordas S, Moran B. Enriched finite elements and level sets for damage tolerance assessment of complex structures. Engng Fract Mech 2006;73:1176-201.

[7] Bordas S, Nguyen PV, Dunant C, Guidoum A, Dang HN. An extended finite element library. Int J Numer Meth Engng 2007;71:703-32.

[8] Chopp DL, Sukumar N. Fatigue crack propagation of multiple coplanar cracks with the coupled extended finite element/fast marching method. Int J Engng Sci 2003;41:845-69.

[9] Civelek MB, Erdogan F. Crack problems for a rectangular plate and an infinite strip. Int J Fract 1982;19:139-59.

[10] Costabel M, Dauge M, Yosibash Z. A quasidual function method for extracting edge stress intensity functions. SIAM J Math Anal $2004 ; 35$ (5):11771202.

[11] Duarte CA. The hp Cloud Method. PhD dissertation, The University of Texas at Austin. Austin, TX, USA, December 1996.

[12] Duarte CA, Babuška I. A global-local approach for the construction of enrichment functions for the generalized fem and its application to propagating three-dimensional cracks. In Leitão VMA, Alves CJS, Duarte CA, editors. ECCOMAS thematic conference on meshless methods. Lisbon, Portugal, 11-14 July 2005. p. 8. 
[13] Duarte CA, Kim D-J. Analysis and applications of a generalized finite element method with global-local enrichment functions. Comput Meth Appl Mech Engng 2008;197(6-8):487-504. http://dx.doi.org/10.1016/j.cma.2007.08.017.

[14] Duarte CA, Babuška I, Oden JT. Generalized finite element methods for three dimensional structural mechanics problems. Comput Struct 2000;77:215-32.

[15] Duarte CA, Hamzeh ON, Liszka TJ, Tworzydlo WW. A generalized finite element method for the simulation of three-dimensional dynamic crack propagation. Comput Meth Appl Mech Engng 2001;190(15-17):2227-62. http://dx.doi.org/10.1016/S0045-7825(00)00233-4

[16] Duarte CAM, Oden JT. Hp clouds - A meshless method to solve boundary-value problems. Technical Report 95-05, TICAM, The University of Texas at Austin, May 1995.

[17] Duarte CAM, Oden JT. Hp clouds - An $h p$ meshless method. Numer Meth Partial Different Eq 1996;12:673-705.

[18] Duarte CAM, Oden JT. An $h p$ adaptive method using clouds. Comput Meth Appl Mech Eng 1996;139:237-62.

[19] Düster A, Niggl A, Rank E. Applying the hp-d version of the fem to locally enhance dimensionally reduced models. Comput Meth Appl Mech Engng 2007;196:3524-33.

[20] Felippa CA. Introduction to finite element methods. Course Notes. Department of Aerospace Engineeing Sciences, University of Colorado at Boulder; 2004 <http://www.colorado.edu/engineering/Aerospace/CAS/courses.d/IFEM.d>.

[21] Fish J. The s-version of the finite element method. Comput Struct 1992;43:539-47.

[22] Fries T-P, Belytschko T. The generalized/extended finite element method: an overview of the method and its applications. Int J Numer Meth Engng 2010:253-304

[23] Gendre L, Allix O, Gosselet P, Comte F. Non-intrusive and exact global/local techniques for structural problems with local plasticity. Comput Mech 2009;44(2):233-45. http://dx.doi.org/10.1007/s00466-009-0372-9.

[24] Gendre L, Allix O, Gosselet P. A two-scale approximation of the Schur complement and its use for non-intrusive coupling. Int J Numer Meth Engng 2011. http://dx.doi.org/10.1002/nme.3142.

[25] Giner E, Sukumar N, Denia FD, Fuenmayor FJ. Extended finite element method for fretting fatigue crack propagation. Int J Solids Struct 2008;45:5675-87.

[26] Giner E, Sukumar N, Tarancón JE, Fuenmayor FJ. An Abaqus implementation of the extended finite element method. Engng Fract Mech 2009;76(3):347-68. http://dx.doi.org/10.1016/i.engfracmech.2008.10.015.

[27] Gravouil A, Moës N, Belytschko T. Non-planar 3d crack growth by the extended finite element and level sets - part II: level set update. Int J Numer Meth Engng 2002;53(11):2569-86.

[28] Gupta V, Kim D-J, Duarte CA. Analysis and improvements of global-local enrichments for the generalized finite element method. Comput Meth Appl Mech Engng, submitted for publication.

[29] Gupta V, Kim D-J, Duarte CA. Extensions of the two-scale generalized finite element method to nonlinear fracture problems. Int J Multisc Comput Engng, submitted for publication.

[30] Kim D-J, Duarte CA, Pereira JP. Analysis of interacting cracks using the generalized finite element method with global-local enrichment functions. J Appl Mech 2008;75(5):051107. http://dx.doi.org/10.1115/1.2936240.

[31] Kim D-J, Pereira JP, Duarte CA. Analysis of three-dimensional fracture mechanics problems: a two-scale approach using coarse generalized FEM meshes. Int J Numer Meth Engng 2010;81(3):335-65. http://dx.doi.org/10.1002/nme.2690.

[32] Kim D-J, Duarte CA, Proenca SP. A generalized finite element method with global-local enrichment functions for confined plasticity problems. Comput Mech, in press. doi:http://dx.doi.org/10.1007/s00466-012-0689-7.

[33] Kim D-J, Duarte CA, Sobh NA. Parallel simulations of three-dimensional cracks using the generalized finite element method. Comput Mech 2011;47(3):265-82. http://dx.doi.org/10.1007/s00466-010-0546-5.

[34] Krause R, Rank E. Multiscale computations with a combination of the h- and p-versions of the finite-element method. Comput Meth Appl Mech Engng 2003;192:3959-83.

[35] Tool command language <http://www.tcl.tk/>.

[36] Lua J, Shi J, Liu P, Collette M. Curvilinear crack growth and remaining life prediction of aluminum weldment using X-FEM. In: 49th AIAA/ASME/ASCE/ AHS/ASC structures, structural dynamics, and materials conference. Schaumburg, IL, USA, 2008. p. 1-19 AIAA $2008-1839$.

[37] Melenk JM, Babuška I. The partition of unity finite element method: Basic theory and applications. Comput Meth Appl Mech Engng 1996;139:289-314.

[38] Moës N, Dolbow J, Belytschko T. A finite element method for crack growth without remeshing. Int J Numer Meth Engng 1999;46:131-50.

[39] Moës N, Gravouil A, Belytschko T. Non-planar 3D crack growth by the extended finite element and level sets - part I: mechanical model. Int J Numer Meth Engng 2002;53(11):2549-68.

[40] Nistor I, Pantalé O, Caperaa S. Numerical implementation of the extended finite element method for dynamic crack analysis. Advan Engng Softw 2008;39(7):573-87. http://dx.doi.org/10.1016/i.advengsoft.2007.06.003.

[41] Oden JT, Duarte CA, Zienkiewicz OC. A new cloud-based $h p$ finite element method. Comput Meth Appl Mech Engng 1998;153:117-26.

[42] Pereira JP, Duarte CA. Computation of stress intensity factors for pressurized cracks using the generalized finite element method and superconvergent extraction techniques. In: Lyra PRM, da Silva SMBA, Magnani FS, Guimaraes LJ do N, da Costa LM, Parente Junior E, editors. XXV Iberian Latin-American congress on computational methods in engineering. Recife, PE, Brazil, November 2004. p. 15. ISBN Proceedings CD: $857409869-8$.

[43] Pereira JP, Duarte CA. Extraction of stress intensity factors from generalized finite element solutions. Engng Anal Bound Elements 2005;29:397-413.

[44] Pereira JP, Duarte CA, Guoy D, Jiao X. Hp-Generalized FEM and crack surface representation for non-planar 3-D cracks. Int J Numer Meth Engng 2009;77(5):601-33. http://dx.doi.org/10.1002/nme.2419.

[45] Pereira JP, Duarte CA, Jiao X, Guoy D. Generalized finite element method enrichment functions for curved singularities in 3D fracture mechanics problems. Comput Mech 2009;44(1):73-92. http://dx.doi.org/10.1007/s00466-008-0356-1.

[46] Pereira JP, Duarte CA, Jiao X. Three-dimensional crack growth with $h p$-generalized finite element and face offsetting methods. Comput Mech 2010;46(3):431-53. http://dx.doi.org/10.1007/s00466-010-0491-3.

[47] Pereira JP, Kim D-J, Duarte CA. A two-scale approach for the analysis of propagating three-dimensional fractures. Comput Mech 2012;49(1):99-121. http://dx.doi.org/10.1007/s00466-011-0631-4.

[48] Python <http://www.python.org/>.

[49] Raju JC, Newman Jr IS. Three dimensional finite-element analysis of finite-thickness fracture specimens. Report TN D-8414, NASA - Langley Research Center, Hampton, VA, May 1977. p. 1-40.

[50] Raju JC, Newman Jr IS. Stress-intensity factors for a wide range of semi-elliptical surface cracks in finite-thickness plates. Engng Fract Mech 1979;11:817-29.

[51] Rank E, Krause R. A multiscale finite element method. Comput Struct 1997;64:139-44.

[52] Shi GH. Manifold method of material analysis. In: Transactions of the 9th army conference on applied mathematics and computing, report no. 92-1 (US Army Research Office, 1991); 1991.

[53] Shi J, Lua J, Waisman H, Liu P, Belytschko T, Sukumar N, et al. X-FEM toolkit for automated crack onset and growth prediction. In: 49th AIAA/ASME/ ASCE/AHS/ASC structures, structural dynamics, and materials conference. Schaumburg, IL, USA, April 2008. p. 1-22. AIAA 2008-1763.

[54] Shi J, Chopp D, Lua J, Sukumar N, Belytschko T. Abaqus implementation of extended finite element method using a level set representation for threedimensional fatigue crack growth and life predictions. Engng Fract Mech 2010;77(14):2840-63. http://dx.doi.org/10.1016/i.engfracmech.2010.06.009.

[55] Sukumar N, Prevost J-H. Modeling quasi-static crack growth with the extended finite element method part I: computer implementation. Int J Solids Struct 2003;40:7513-37.

[56] Sukumar N, Moës N, Moran B, Belytschko T. Extended finite element method for three-dimensional crack modelling. Int J Numer Meth Engng 2000;48(11):1549-70. 
[57] Sukumar N, Chopp DL, Moran B. Extended finite element method and fast marching method for three-dimensional fatigue crack propagation. Engng Fract Mech 2003;70(11):29-48.

[58] Szabo BA, Babuška I. Computation of the amplitude of stress singular terms for cracks and reentrant corners. In: Cruse TA, editor. Fracture mechanics: 19th symposium, ASTM STP 969. Southwest Research Institute, San Antonio, TX, 1988. p. 101-24.

[59] Terada K, Asai M, Yamagishi M. Finite cover method for linear and nonlinear analyses of heterogeneous solids. Int J Numer Meth Engng 2008;58:1321-46.

[60] Tian R, Yagawa G. Generalized nodes and high-performance elements. Int J Numer Meth Engng 2005;64:2039-71.

[61] Tian R, Matsubara H, Yagawa G. Advanced 4-node tetrahedrons. Int J Numer Meth Engng 2006:68:1209-31.

[62] Walters MC, Paulino GH, Dodds Jr RH. Stress-intensity factors for surface cracks in functionally graded materials under mode-I thermomechanical loading. Int J Solids Struct 2004;41:1081-118.

[63] Wyart E, Coulon D, Duflot M, Pardoen T, Remacle J-F, Lani F. A substructured FE-shell/XFE-3D method for crack analysis in thin-walled structures. Int J Numer Meth Engng 2007;72:757-79.

[64] Xu Y, Yuan H. Computational analysis of mixed-mode fatigue crack growth in quasi-brittle materials using extended finite element methods. Engng Fract Mech 2009;76(2):165-81. http://dx.doi.org/10.1016/j.engfracmech.2008.08.011.

[65] Xu Y, Yuan H. Computational modeling of mixed-mode fatigue crack growth using extended finite element methods. Int J Fract 2009;159(2):151-65. http://dx.doi.org/10.1007/s10704-009-9391-y. 